\title{
TOP2A and CENPF are synergistic master regulators activated in cervical cancer
}

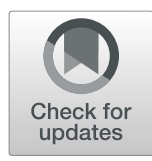

Beiwei $\mathrm{Yu}^{1+}$, Long Chen ${ }^{2 \dagger}$, Weina Zhang ${ }^{3 \dagger}$, Yue Li ${ }^{4}$, Yibiao Zhang ${ }^{5}$, Yuan Gao ${ }^{3}$, Xianlin Teng ${ }^{4}$, Libo Zou ${ }^{6}$, Qian Wang ${ }^{7}$, Hongtao Jia ${ }^{7}$, Xiangtao Liư ${ }^{7}$, Hui Zheng ${ }^{7}$, Ping Hou ${ }^{3}$, Hongyan $\mathrm{Yu}^{3}$, Ying Sun ${ }^{3}$, Zhiqin Zhang ${ }^{8}$, Ping Zhang ${ }^{3}$ and Liqin Zhang ${ }^{1,4^{*}}$

\begin{abstract}
Background: Identification of master regulators (MRs) using transcriptome data in cervical cancer (CC) could help us to develop biomarkers and find novel drug targets to fight this disease.

Methods: We performed differential expression (DE) analyses of public microarray and RNA-seq transcriptome data of CC and normal cervical tissues (N). Virtual Inference of Protein activity by Enriched Regulon analysis (VIPER) was used to convert the DE outcomes to differential activity (DA) signature for MRs. Synergy analysis was conducted to study synergistic effect of MR-pairs. TCGA and microarray data were used to test the association of expression of a MR and a clinical feature or a molecular feature (e.g. somatic mutations). Various bioinformatic tools/websites (DAVI D, GEPIA2, Oncomine, cBioPortal) were used to analyze the expression of the top MRs and their regulons.

Results: Ten DE and 10 DA signatures were generated for CC. Two MRs, DNA topoisomerase II alpha (TOP2A) and centromere protein $\mathrm{F}$ (CENPF) were found to be up-regulated, activated and synergistic in CC compared to $\mathrm{N}$ across the 10 datasets. The two MRs activate a common set of genes (regulons) with functions in cell cycle, chromosome, DNA damage etc. Higher expression of CENPF was associated with metastasis. High expression of both MRs is associated with somatic mutation of a set of genes including tumor suppressors (TP53, MSH2, RB1) and genes involved in cancer pathways, cell cycle, DNA damage and repair. The magnitude of up-regulation and the absolute expression level of both MRs in CC are significantly higher compared to many other cancer types.

Conclusion: TOP2A and CENPF are a synergistic pair of MRs that are overexpressed and activated in CC. Their high expression is correlated with some prognosis features (e.g. metastasis) and molecular features (e.g. somatic mutations) and distinctly high in CC vs. many other cancer types. They may be good biomarkers and anticancer drug targets for CC.
\end{abstract}

Keywords: Cervical cancer, Master regulator, Transcriptome, Differential expression, Differential activity

\footnotetext{
* Correspondence: 1939277107@qq.com

†Beiwei Yu Long Chen and Weina Zhang are co-first authors.

'Department of Laboratory, Hangzhou Jianggan District People's Hospital,

Hangzhou, Zhejiang, China

${ }^{4}$ Department of Laboratory, Jinhua People's Hospital, Jinhua, Zhejiang, China

Full list of author information is available at the end of the article
}

(C) The Author(s). 2020 Open Access This article is licensed under a Creative Commons Attribution 4.0 International License, which permits use, sharing, adaptation, distribution and reproduction in any medium or format, as long as you give appropriate credit to the original author(s) and the source, provide a link to the Creative Commons licence, and indicate if changes were made. The images or other third party material in this article are included in the article's Creative Commons licence, unless indicated otherwise in a credit line to the material. If material is not included in the article's Creative Commons licence and your intended use is not permitted by statutory regulation or exceeds the permitted use, you will need to obtain permission directly from the copyright holder. To view a copy of this licence, visit http://creativecommons.org/licenses/by/4.0/ The Creative Commons Public Domain Dedication waiver (http://creativecommons.org/publicdomain/zero/1.0/) applies to the data made available in this article, unless otherwise stated in a credit line to the data. 


\section{Background}

Cervical cancer (CC) is the fourth most common female malignancy worldwide. More than half a million women are diagnosed with cervical cancer and the disease results in over 300,000 deaths worldwide each year [1, 2]. Extended understanding of the gene expression programs, especially the master regulators (MRs) in CC, will help us to fight this devastating disease.

Since early 2000s, microarrays have been used to profile transcriptome of cervical cancer tissues. Further, the application of RNA-sequencing (RNAseq) in recent years has rapidly generated large amount of data on the gene expression landscape of this disease. Differential expression (DE) analysis is the most common and straightforward analysis for a gene expression dataset. Through DE analysis, a set of highly up-regulated and down-regulated genes can be identified by comparing two groups of samples (e.g. cancer vs. normal). However, gene expression is highly dynamic, and the expression quantification may depend on the techniques (e.g. different platforms of microarray, or RNAseq), making the cross-dataset comparison difficult. Moreover, the top regulated gene may not represent the key genes or the MRs under a biological system. The activities of MRs cannot be directly measured by microarray or RNA-seq because these techniques only measure RNA expression level and do not consider protein level and protein activity changes by post-translational modifications. Fortunately, MR activity can be inferred by its regulons (genes downstream and regulated by of the MR) through the "Virtual Inference of Protein activity by Enriched Regulon analysis" (VIPER) [3], which takes the DE data and an context-specific gene regulatory network (interactome) as inputs.

The presence of MRs in various cancers has been well-documented, whose coordinated activity within tightly regulated modules (tumor checkpoints) are strictly necessary for tumor state initiation and maintenance [3]. To identify the top MRs in $\mathrm{CC}$, we initially collected 10 transcriptome datasets from Gene Expression Omnibus (GEO) [4] with $\mathrm{CC}$ and normal cervical tissue $(\mathrm{N})$ samples. We performed gene expression analysis using $\mathrm{CC}$ compared to $\mathrm{N}$ and generated 10 DE signatures. Then VIPER was conducted to convert the $10 \mathrm{DE}$ signatures to 10 differential activity (DA) signatures for MRs. We further analyzed the DA-DE consistency, the synergy of $M R$ pairs and identified TOP2A-CENPF as a synergistic MR pair in CC. Both MRs showed increased activity and expression. We further studied the common regulons of the two MRs, the links between the expression of the two MRs and clinical or molecular features of CC. Finally, we compared the expression of them in $\mathrm{CC}$ vs. other cancer types.

\section{Methods \\ GEO data collection}

Data were collected through searching Gene Expression Omnibus (GEO) [4] for microarray or RNA-seq data for gene expression profiling using key word "cervical cancer". All datasets were manually inspected to select datasets with both cervical cancer (CC) tissue samples and normal (N) cervical tissue samples. To eliminate the potential noise from small number of samples, a minimum of 5 samples per group was required for each dataset. A summary of all data used can be found in Table 1 .

\section{Gene expression analysis of microarray data from GEO database}

Gene expression of microarray data were computed using the GEO2R tool (https://www.ncbi.nlm.nih.gov/ geo/geo2r/). The CC and $\mathrm{N}$ samples within the same dataset were compared. Normalized expression values of genes or probes were visualized within GEO2R tool as a boxplot. Obvious outliers (samples with distribution of normalized values very different from the rest of samples) were excluded from the dataset for further analysis.

Table 1 Datasets used in this study

\begin{tabular}{|c|c|c|c|c|c|c|}
\hline Data Name & GEO ID & \#Tumor & \#Normal & Platform & Dataset type & $\operatorname{Ref}^{a}$ \\
\hline Biewenga_2008 & GSE7410 & 19 & 5 & Agilent Oligo G4112A & discovery & {$[5]$} \\
\hline Boon_2015 & GSE63514 & 28 & 24 & Affymetrix U133 Plus 2.0 & discovery & {$[6]$} \\
\hline Guardado_2012 & GSE29570 & 45 & 17 & Affymetrix Gene 1.0 ST & discovery & {$[7]$} \\
\hline Medina_2014 & GSE52903 & 55 & 17 & Affymetrix Gene 1.0 ST & discovery & {$[8]$} \\
\hline Zhai_2007 & GSE7803 & 21 & 10 & Affymetrix U133A & discovery & {$[9]$} \\
\hline Li_2018 & GSE107472 & 5 & 5 & RNA-seq & validation & {$[10]$} \\
\hline Pappa_2015 & GSE63678 & 5 & 5 & Affymetrix HG133_A_2.0 & validation & {$[11]$} \\
\hline Pyeon_2007 & GSE6791 & 20 & 8 & Affymetrix U133 Plus 2.0 & validation & {$[12]$} \\
\hline Scotto_2008 & GSE9750 & 33 & 24 & Affymetrix U133A & validation & {$[13]$} \\
\hline Sun_2014 & GSE55940 & 5 & 5 & Glue Grant Transcriptome 3.0 & validation & [14] \\
\hline
\end{tabular}

${ }^{\mathrm{a}}$ Ref. references 
Statistical test results for all probes were downloaded. Moderated $t$-statistic were used as the DE measurement for the dataset. If one gene has multiple probes, only the one with highest absolute $t$ value was used. All probes without assigned gene ID were discarded. For dataset Biewenga_2008 [5], the expression values for an individual gene/probe for all samples were extracted using the "Profile graph" function in GEO2R tool. The expression differences between different sample types (ie. N, Normal; C, tumour without lymph node metastasis; $M$, tumour with lymph node metastasis) were analyzed using Wilcoxon Rank-Sum test.

\section{Gene expression analysis of RNA-seq data from GEO database}

Fastq files were downloaded from Sequence Read Archive (SRA, https://www.ncbi.nlm.nih.gov/sra/, [15]) database using SRA Toolkit (https://ncbi.github.io/sra-tools/ ). Reads were mapped to human reference genome (hg19) using STAR (2.6.0a) [16]. Reads with mapping quality score $(\mathrm{MAPQ})<10$ or those with $>5$ mismatches in $100 \mathrm{bp}$ aligned region were discarded. Reads mapped to coding sequence (CDS) region of Refseq-defined genes were used to quantify gene expression. For nonprotein-coding transcripts, reads mapped to all exons were used to quantify gene expression. For genes with multiple transcripts, only the transcript with the largest number of reads (sum for all samples) was used. DESeq2 [17] was used for differential expression (DE) analysis comparing CC vs. $\mathrm{N}$ samples. The DE measurement for RNA-seq is $-\mathrm{S} \times \log 10$ (adjusted $P$-value), in which $\mathrm{S}$ is the sign indicating the direction of regulation $(+1$ for upregulation and - 1 for downregulation).

\section{Master regulator (MR) analysis using VIPER}

Virtual inference of protein activity by enriched regulon analysis (VIPER) [3] was conducted to infer the master regulator differential activities (DA) from the DE data. The "msviper" function in R package "viper" was used as the program to run VIPER analysis. VIPER takes a DE signature and a regulatory network as inputs. Contextspecific regulatory networks "aracne.networks" [18] were downloaded from Bioconductor. Human cervical squamous carcinoma context-specific ARACNe interactome "reguloncesc" was used as the "network" in this study. Parameter "pleiotropy" was set to TRUE to correct for the effects of target overlap between different MRs. VIPER outputs $P$-values and normalized enrichment scores (NES) which represents the DA signature of MRs.

\section{MR categories}

These 5838 MRs in interactome "reguloncesc" were categorized into three groups based on their function annotation in gene ontology (GO) database (http:// geneontology.org/): (1) 1588 transcriptional factors (TFs): genes annotated in the GO Molecular Function database GO:0003700 (DNA binding transcription factor activity), or GO:0003677 (DNA binding) and GO: 0030528 (Transcription regulator activity), or GO: 0003677 and GO:0045449 (Regulation of transcription); (2) 280 transcriptional co-factors (TFcoFac): genes not in TF and annotated as GO:0003712 (transcription cofactor activity), GO:0030528 or GO:0045449; (3) 3883 in signal pathways (SigPathway): genes not in TF or TFcoFac and annotated in the GO Biological Process database as GO:0007165 (signal transduction) or in the GO Cellular Component database as GO:0005622 (intracellular) or GO:0005886 (plasma membrane); 87 MRs were not annotated in these three categories.

\section{Selecting common top MRs in CC}

The 10 GEO datasets (Table 1) were randomly assigned to two groups: 5 discovery datasets and 5 validation datasets. To select top common MRs in CC, the top $\mathrm{N}$ $(N=50,100,200,300)$ most activated MRs and top $\mathrm{N}$ suppressed MRs in each one of five discovery datasets were selected. The common activated and suppressed MRs were the common ones in the top N MRs from all the five discovery datasets. Whether these common top MRs were also significant in the validation datasets were examined by comparing the DA values of the common MRs vs. all the other MRs using Wilcoxon Rank-Sum test. We also require that these top MRs have significant and consistent DE change in the discovery datasets. With another requirement that $\mathrm{DE}>3$ or $\mathrm{DE}<-3$ for when the DA is positive or negative respectively, the resulting MRs are called DA-DE consistent MRs. In this study, the results for $N=100$ were presented in the main text and results for all Ns $(N=50,100,200,300)$ are provided in the supplementary Table 1.

\section{Synergy analysis of MR pairs}

Synergy of two MRs was to test whether their common regulons have a more extreme $\mathrm{DE}$ signature than the rest of regulons. Among the DA-DE consistent MRs, all pairs of MRs were considered for synergy analysis. For a given pair of MRs (denoted here as MR1 and MR2), their regulons were extracted from the interactome "reguloncesc" in regulatory networks "aracne.networks" [18]. Then the common regulons for both MRs were selected. Only when MR1 and MR2 have > 10 and > 10\% common regulons among the regulon number in both MR1 and MR2, the synergy analysis was conducted. All regulons belong to MR1 or MR2 were separated to three groups: common, MR1-unique and MR2-unique. For each DE dataset, two statistical tests were performed: common vs. MR1-unique for MR1 regulons and common vs. MR2unique for MR2 regulons. The Expression Contribution 
to Activity (ECA) values of all regulons were calculated for each MR and each DE dataset. ECA = DE $x$ MOR, in which DE is the differential expression value and MOR is the mode of regulation value of a MR-regulon pair from the interactome "reguloncesc". MOR indicates the sign of the association between a MR (regulator) and a regulon (target gene) and ranges between -1 and +1 , with positive and negative value indicating positive and negative regulation respectively. The ECA distribution of common regulons were compared with MR1-unique or MR2-unique regulons, using Wilcoxon Rank-Sum test (one tailed), in which the alternative hypothesis of the test is "greater" and "less" when the sign of the DA of the MR in the DE dataset is positive and negative respectively. A synergistic MR pair will show significant higher ECA values for common regulons than other regulons if the MR is activated (positive DA value) and vice versa.

\section{Network visualization of MRs and regulons}

The MR and regulon network were visualized using Cytoscape 3.7.1 [19]. A node is either a MR or a regulon. An edge is a MR-regulon relationship. Node color represents the average DE scores of a gene (a MR or a regulon) in the 10 datasets with red and blue indicating upregulation and down-regulation respectively. Edge color represent the MOR of a MR and a regulon relationship with red and blue indicating positive and negative regulation respectively.

\section{DAVID analysis}

The function annotation analysis for a given gene list was conducted using the Database for Annotation, Visualization and Integrated Discovery (DAVID) v6.8 (https://david.ncifcrf.gov/) [20]. The gene symbols of a gene list were submitted to the DAVID webserver. "OFFICIAL_GENE_SYMBOL" was chosen to map the genes to the IDs in the DAVID database. The annotations of genes were limited to "Homo sapiens" only. The background gene list was also set to "Homo sapiens". The "Functional Annotation Clustering" function of the website was taken. Several gene annotation databases were included: UP_KEYWORDS, UniProt Keywords; UP_ SEQ_FEATURE, UniProt sequence feature; GOTERM_ BP_DIRECT, Gene ontology biological process; GOTERM_CC_DIRECT, Gene ontology cellular component; KEGG_PATHWAY, KEGG pathway. The results were downloaded and certain generic annotation terms with total gene number ("Pop Hits") > 1000 were removed.

\section{TCGA data}

The gene expression data for individual genes for CC samples in The Cancer Genome Atlas (TCGA) [21] was retrieved from TSVdb [22]. The RSEM (RNA-Seq by Expectation Maximization) values were used. The overall survival (OS) data was also retrieved from TSVdb. Other clinical and molecular features (e.g. Somatic mutation data) data were retrieved from the supplementary Table 1 and 2 of Cancer Genome Atlas Research Network et al., 2017 [23]. The loss-of-function (LOF) mutations include nonsense, frame shift and splicing mutations. A sample with both a missense mutation and a LOF mutation of a gene is classified as LOF. The description of each clinical or molecular feature can be found in the "features list" tab in the supplementary Table 1 of Cancer Genome Atlas Research Network et al., 2017 [23]. The features were separated to categorical or numerical data. For each categorical feature, only the sample groups with $>5$ samples were analyzed. The expression values of a MR for different groups of a categorical feature were compared using Wilcoxon Rank-Sum test. All gene somatic mutation data were treated as categorical data with three groups: no mutation (None), missense mutation and LOF mutation. Rather than setting a minimal sample number threshold to filter the data, we selected all genes with missense or LOF mutations having expression of a MR different than the None group with $P<0.1$. For each numerical feature, the Spearman correlation was computed to test the significance of the correlation of the expression of a MR and the feature.

For survival analysis, all patients with OS data were divided into two groups based on the 80th percentile value of the expression of a gene. The survival curve was drawn using the R package "survminer" (https://cran.rproject.org/web/packages/survminer/). P-value is calculated based on the log-rank test.

\section{Network visualization of association of MRs and genes with somatic mutations}

The MR and somatic mutation gene network were visualized using Cytoscape 3.7.1 [19]. A node is either a MR or a gene with somatic mutations (SMG) in TCGA CC samples. An edge is a MR-SMG relationship. Edge color represents the direction of a MR-SMG relationship with red and blue indicating that samples with a SMG with mutations associated with high and low expression of a MR respectively. The arrowhead shape represents the type of a mutation with an open square and a solid diamond representing a missense mutation and a LOF mutation respectively.

\section{GEPIA2 analysis}

Gene Expression Profiling Interactive Analysis 2 (GEPI A2) is a web server for analyzing the RNA sequencing expression data of 9736 tumors and 8587 normal samples from the TCGA and the GTEx projects (http:// gepia2.cancer-pku.cn) [24]. We analyzed the expression 
of the top MRs in cancer and normal tissues for 33 cancer types using the "Dot plot" function of GEPIA2. The expression of a gene is plotted in $\log 2(\mathrm{TPM}+1)$ scale, in which TPM is the Transcript Per Million value. Analysis of variance (ANOVA) was used to test the statistical significance of the difference of expression in tumor tissues vs. paired normal tissues.

\section{Oncomine analysis}

Oncomine is a bioinformatics platform aimed at collecting, standardizing and analyzing cancer transcriptome microarray data (http://www.oncomine.org) [25]. We used Oncomine 4.5 to analyze the expression of top MRs (CENPF and TOP2A) in different cancers types. All datasets were filtered using the gene symbol, "Analysis Type $=$ Cancer vs. Cancer Analysis" and "Cancer Type = cervical cancer". The "Bittner Multi-cancer" dataset was returned as the top significant dataset. "Bittner Multicancer" is from the Expression Project for Oncology (expO) which contains 1911 various tumor samples analyzed on Affymetrix U133 Plus 2.0 microarrays (unpublished, GEO ID, GSE2109). The $\log 2$ median-centered intensity was used as gene expression value. The expression difference of a MR in cervical cancer vs. other cancer types was tested using Student's $t$-test.

\section{The human protein atlas}

The immunohistochemistry of cervical cancer tissue, protein subcellular localization and cell cycle expression pattern information were extracted from the "PATHOLOGY" and "CELL" tab of The Human Protein Atlas (http://www.proteinatlas.org) [26]. The cell cycle dependency expression data for a protein was analyzed using a custom assay by staining of U-2 OS FUCCI cells. The FUCCI cells, Fluorescence Ubiquitination Cell Cycle Indicator, are cells tagged with different fluorescent proteins fused to two different cell cycle regulators Cdt1 (expressed in G1 phase) and Geminin (expressed in S and G2 phases) that allows cell cycle monitoring. When both proteins are present, the overlay of the images appear in yellow marking the G1/S transition.

\section{OncoPrint visualization using cBioPortal}

OncoPrints are compact means of visualizing distinct genomic alterations, including somatic mutations, copy number alterations, and mRNA expression changes across a set of cases. OncoPrint was generated using cBioPortal [27].

\section{Protein-protein association analysis using STRING}

STRING [28] was used to study the protein-protein association of TOP2A, CENPF and their common regulons with average DE score (DE.average) $>5$. Only associations experimentally determined, from curated database or from text mining were shown. The kmeans clustering method with the number of clusters $=3$ was used for the clustering analysis.

\section{DepMap analysis}

The correlation analysis of gene expression and promoter methylation for TOP2A and CENPF was performed using the Data Explorer tool of DepMap [29].

\section{Expression signature comparison with the connectivity map (CMap) using CLUE}

The Connectivity Map (CMap) [30] is a database developed by the Broad Institute with $>1$ million expression signatures of cells responding to chemical, genetic, and disease perturbations. CLUE (https://clue.io/) [30] is a cloud-based software platform for the analysis of perturbational datasets of CMap. QUERY is a tool in CLUE to find positive and negative connections between the gene expression signature of interest and all the signatures in CMap. We used TOP2A, CENPF and their common regulons with average DE score (DE.average) $>5$ as input upregulated gene list and used QUERY tool to query the CMap database. The result included connectivity score matrix of 8969 perturbations in 9 cell types. The connectivity score is a standardized measure ranging from - 100 to 100 with a positive score indicating a similarity between a given perturbagen's signature and that of the query, while a negative score indicating an opposing signature.

\section{Statistical test}

All statistical tests and plotting were performed in $\mathrm{R}$ 3.5.2 (https://www.r-project.org/) unless otherwise mentioned.

\section{Results}

\section{Identification of top MRs in CC}

To identify the top MRs in CC, a collection of 10 datasets were selected from GEO database (Table 1). Each dataset has at least $5 \mathrm{CC}$ samples and at least 5 normal cervical tissues as controls. Alltogether, $236 \mathrm{CC}$ samples and 120 normal tissues were analyzed. The DE analysis was performed in each of the 10 datasets, resulting in 10 DE signatures. Using VIPER [3], we converted each DE signature of all genes to a DA signature of 5838 MRs (Fig. 1a-c, supplementary Table 1). We sought to use a discovery-and-validation approach (Fig. 1b-c) to identify top MRs. The 10 datasets were randomly assigned to a discovery group and a validation group each include 5 datasets (Table 1). Using the top 100 most activated and top 100 most suppressed MRs in each of the 5 discovery DA signatures, we identified 18 common activated and 3 common suppressed MRs (Fig. 2a left). The 18 and 3 MRs were found to be also significantly enriched in the 


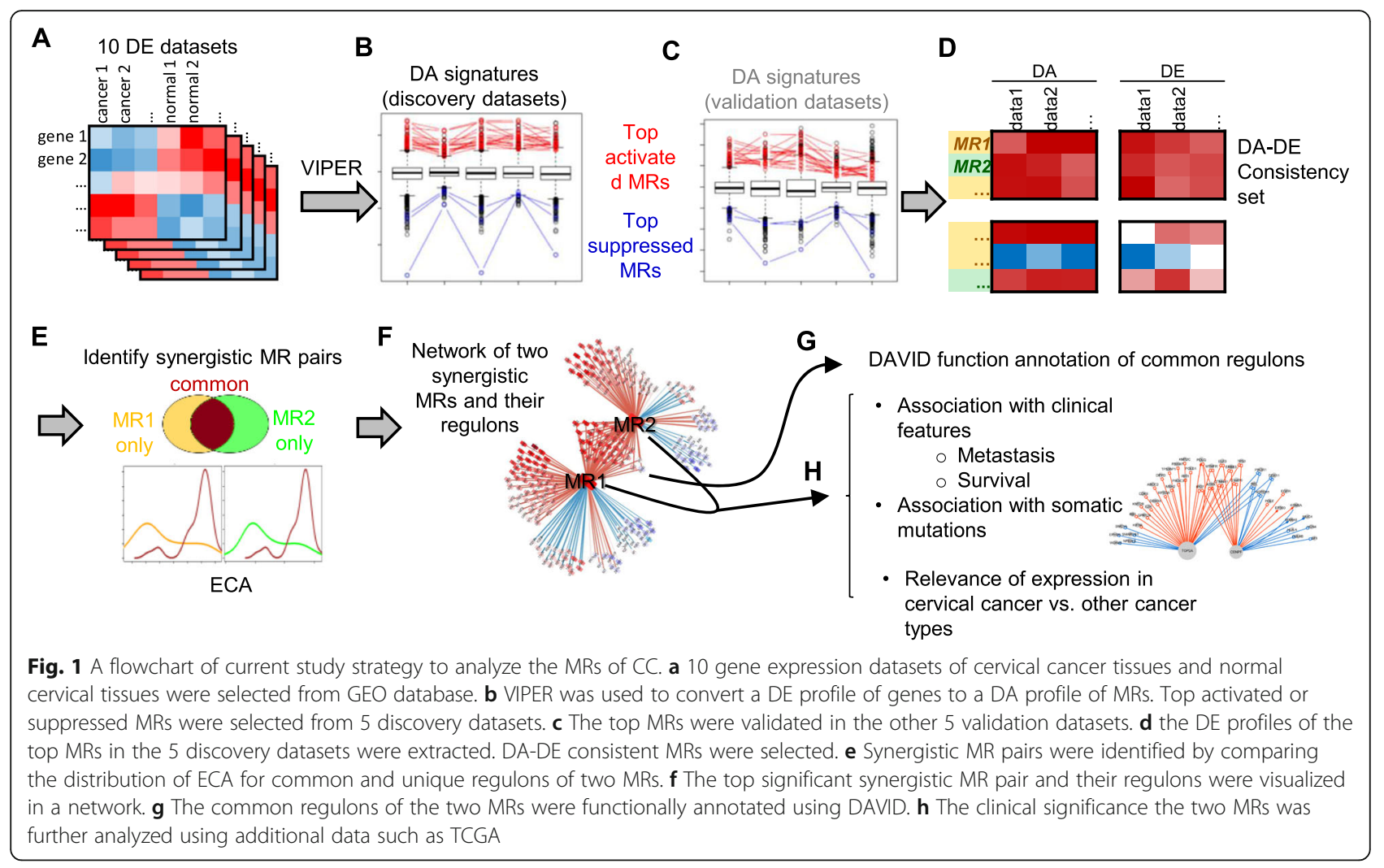

top activated and suppressed MRs, respectively in the 5 validation datasets (Fig. 2a right), indicating that these MRs are robust top MRs in CC. Next, we compared the DA and DE signatures of the 18 and 3 MRs (Fig. 1d) using the 5 discovery datasets and selected 12 MRs which have consistent DA and DE profiles. All the 12 MRs are activated and up-regulated (Fig. 2b). The 12 MRs contain four transcriptional factors or co-factors (GMNN, DNMT1, PSMC3IP, FOXM1) and eight involved in signal pathways (TOP2A, RACGAP1, CENPF, MCM6, DEPDC1, RAD51, TYMS, CCNA2) (Fig. 2 b).

\section{Identification of synergistic MRs TOP2A and CENPF in CC}

Next, we sought to identify synergistic MR-pairs in CC, i.e., the two MRs with their common regulons being more extremely regulated than regulons unique to each of them (Fig. 1e-f). From the 12 DA-DE consistent MRs, four pairs have at least an overlap of 10 regulons and $10 \%$ of regulons (Fig. 3a). The synergy test was conducted using the ECA values of common regulons vs. MR1- or MR2-unique regulons using Wilcoxon RankSum test for the 10 datasets (Fig. 3a-b). MR pair TOP2A and CENPF was found to be highly significant in all the 10 datasets ( $P$-value range from $7 \mathrm{E}-3$ to $1 \mathrm{E}-10)$. The common regulons of them are all positively regulated $(\mathrm{MOR}>0)$ by both MRs and were more up-regulated than the rest of regulons (Fig. 4a). There are all together
34 common regulons of TOP2A and CENPF (supplementary Table 2,3). Most of them (32) are upregulated in all the 10 datasets with an average $\mathrm{DE}>2$ (Fig. 4b, supplementary Table 2). A functional annotation of the common regulons showed that their encoded proteins are significantly associated with (1) mitosis, cell cycle, cell division; (2) kinesin-motor, microtubule-based movement, kinesin complex; (3) chromosome, sister chromatid cohesion, centromere; (4) DNA damage, DNA repair (Fig. 4c, supplementary Table 4).

\section{Clinical implications of expression of TOP2A and CENPF in CC} Next, we explored the question whether the expression of TOP2A and CENPF have any clinical implications. We used Biewenga_2008 (Table 1) microarray data which has 5 normal cervical tissues $(\mathrm{N}), 19$ tumour samples without lymph node metastasis $(C)$ and 16 tumour samples with lymph node metastasis (M) (supplementary Table 5). As expected, both CENPF and TOP2A expression are significantly $(P<0.01)$ higher in $C$ vs. N. Interestingly, both $C E N P F$ and the average of the two genes showed higher expression $(P<0.05)$ in $\mathrm{M}$ vs. C. (Fig. $5 \mathrm{a})$. The median expression of TOP2A is also higher in $\mathrm{M}$ vs. $C$, although the $P$-value is not significant. The expression of CENPF and TOP2A is highly correlated (Pearson correlation coefficient $=0.79$ ) in all the samples. These 

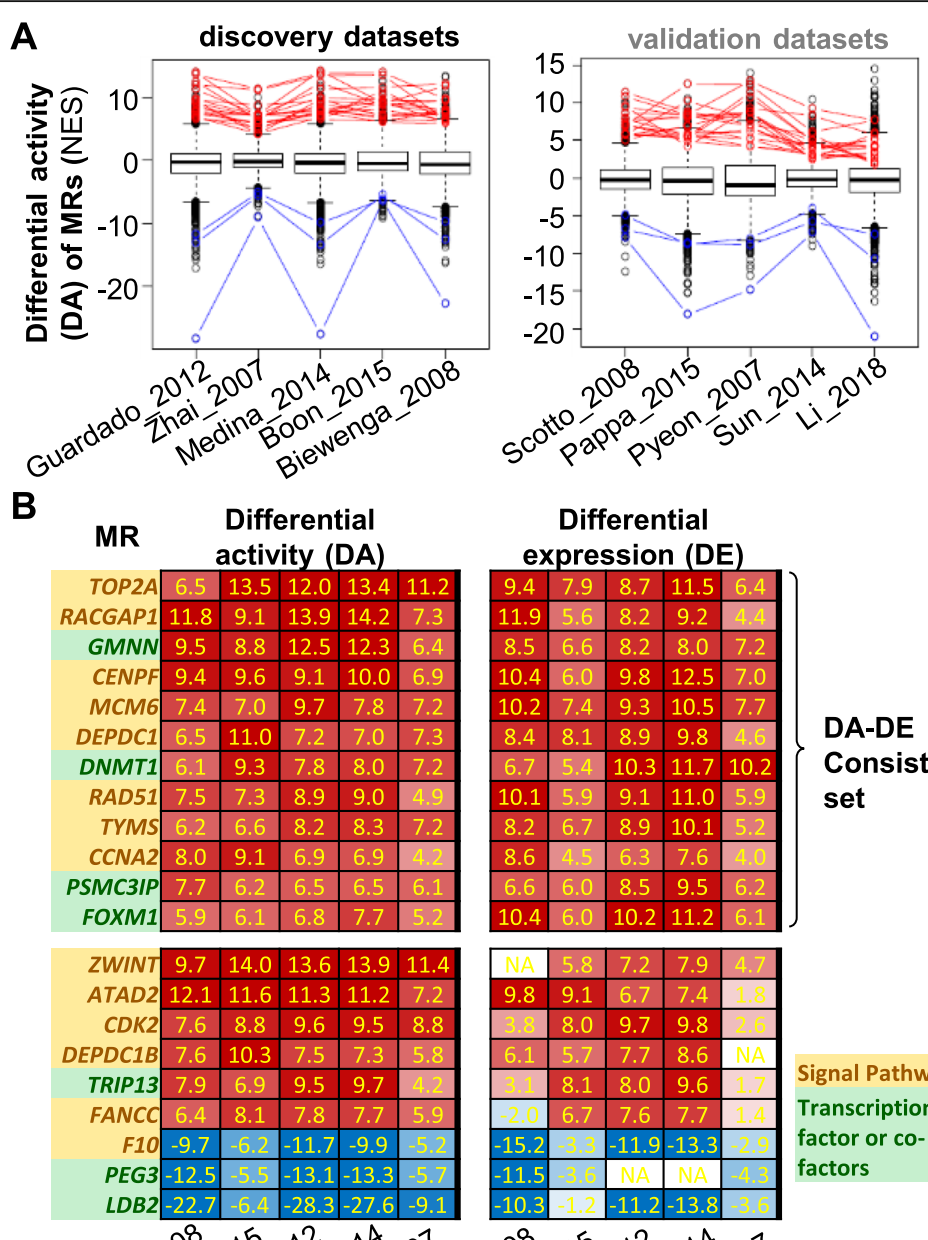

\section{Differential} expression (DE)

\section{\begin{tabular}{c|c|c|c|c|}
9.4 & 7.9 & 8.7 & 11.5 & 6.4 \\
\hline 11.9 & 5.6 & 8.2 & 9.2 & \\
\hline
\end{tabular}}

\begin{tabular}{|c|c|c|c|c|}
\hline 11.9 & 5.6 & 8.2 & 9.2 & 4.4 \\
\hline 8.5 & 6.6 & 8.2 & 8.0 & 7.2 \\
\hline
\end{tabular}

\begin{tabular}{|l|l|l|l|l|}
\hline 8.5 & 6.6 & 8.2 & 8.0 & 7.2 \\
\hline 10.4 & 6.0 & 9.8 & 12.5 & 7.0 \\
\hline
\end{tabular}

\begin{tabular}{|c|c|c|c|c|}
\hline 10.4 & 6.0 & 9.8 & 12.5 & 7.0 \\
\hline 10.2 & 7.4 & 9.3 & 10.5 & 7.7 \\
\hline
\end{tabular}

\begin{tabular}{|c|c|c|c|c|}
\hline 10.2 & 7.4 & 9.3 & 10.5 & 7.7 \\
\hline 8.4 & 8.1 & 8.9 & 9.8 & 4.6 \\
\hline
\end{tabular}

\begin{tabular}{|l|l|l|l|l|}
\hline 8.4 & 8.1 & 8.9 & 9.8 & 4.6 \\
\hline 6.7 & 5.4 & 10.3 & 11.7 & 10.2 \\
\hline
\end{tabular}

\begin{tabular}{c|c|c|c|c}
\hline 8.4 & 8.1 & 8.9 & 9.8 & 4.6 \\
\hline 6.7 & 5.4 & 10.3 & 11.7 & 10.2 \\
\hline 10.1 & 5.9 & 9.1 & 11.0 & 5.9 \\
\hline
\end{tabular}

\section{DA-DE}

\begin{tabular}{|c|c|c|c|c|}
\hline 10.1 & 5.9 & 9.1 & 11.0 & 5.9 \\
\hline
\end{tabular}

\section{Consistency}

\begin{tabular}{|c|c|c|c|c|}
\hline 8.2 & 6.7 & 8.9 & 10.1 & 5.2 \\
\hline 8.6 & 4.5 & 6.3 & 7.6 & 4.0 \\
\hline
\end{tabular}

set

\begin{tabular}{|l|l|l|l|l}
8.2 & 6.7 & 8.9 & 10.1 & 5 \\
\hline 8.6 & 4.5 & 6.3 & 7.6 \\
\hline 6.6 & 6.0 & 8.5 & 9.5 & 6 \\
\hline
\end{tabular}

\begin{tabular}{|l|l|l|l|l|}
\hline 6.6 & 4.5 & 6.3 & 7.6 & 4.0 \\
\hline 6.6 & 6.0 & 8.5 & 9.5 & 6.2 \\
\hline
\end{tabular}

\begin{tabular}{|c|c|c|c|c|}
\hline 10.4 & 6.0 & 10.2 & 11.2 & 6.1 \\
\hline
\end{tabular}

\section{.}

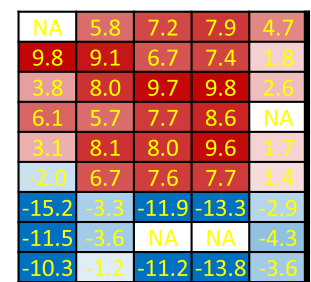

Signal Pathway

Transcriptional

factor or co-

factors

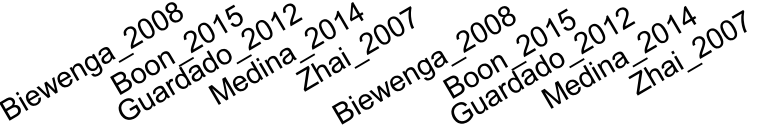

Fig. 2 Identification of top MRs in CC. a Left, Top 100 activated MRs and 100 suppressed MRs were selected within each of the five discovery datasets. The common 18 activated and 3 suppressed MRs were identified and shown as red and blue in the NES boxplot. Right, the same 18 and 3 MRs were shown in the NES boxplot of other 5 validation datasets. The NES value distribution of the 18 and 3 MRs compared to the rest of MRs were tested using Wilcoxon Rank-Sum test. The P-values are 2e-13, 2e-13, 2e-13, 2e-13, 2e-13 for the 18 activated MRs for discovery set; 1e-03, 1e-03, 1e$03,1 \mathrm{e}-03,1 \mathrm{e}-03$ for the 3 suppressed MRs for the discovery set; 1e-13, 4e-13, 1e-12, 3e-12, 9e- 11 for the 18 activated MRs for validation set; $2 \mathrm{e}$ $-03,1 e-03,1 e-03,2 e-03,1 e-03$ for the 3 suppressed MRs for the validation set. $\mathbf{b}$ The DA (NES scores) and DE ( $t$-scores) matrix of the 21 MRs (18 activated and 3 suppressed) in the 5 discovery datasets. DA-DE Consistency set was selected using |DE| value> 3 and same sign as DA in all five datasets. MRs in signal pathway or transcription factors were highlighted in orange and green background respectively

observations were confirmed using the TCGA data (RNA-seq), which has $3 \mathrm{~N}$ samples, 304 C samples and $2 \mathrm{M}$ samples (Fig. 5b, supplementary Table 6). From a survey of additional 21 clinical or molecular features (including 14 categorical and 7 numerical features, supplementary Figure 1, 2), we found that clinical feature lymphovascular space invasion (LVSI) and lymphnodes $(\mathrm{LN})$ positive is associated with higher $(P<0.05)$ expression of TOP $2 A$ or the average expression of the two genes in CC tissues (Fig. 5c, supplementary Table 6). The samples with molecular feature APOBEC mutagenesis (Low) has higher expression of CENPF or the average of the two genes $(P<0.05)$ than the samples without
APOBEC mutations (No) (Fig. 5d, supplementary Table 6). The OS of samples with high expression of CENPF is slightly poorer than those with lower expression, although the $P$-value did not reach significance level $(P=$ 0.15 , supplementary Figure 3$)$. Among all the numerical features, only the sample purity computed by ABSOLUTE (Purity_Absolute) showed positive correlation with the expression of CENPF $(P=0.03)$ and TOP $2 A$ $(P=3 \mathrm{E}-5) \quad$ (supplementary Figure 2$)$, confirming the finding that these genes are highly up-regulated in $\mathrm{CC}$ compared to $\mathrm{N}$ tissues. Other features, including race, human papillomavirus (HPV) status or type, clinical stage, EMT score, mutation rate, etc. do not correlate 


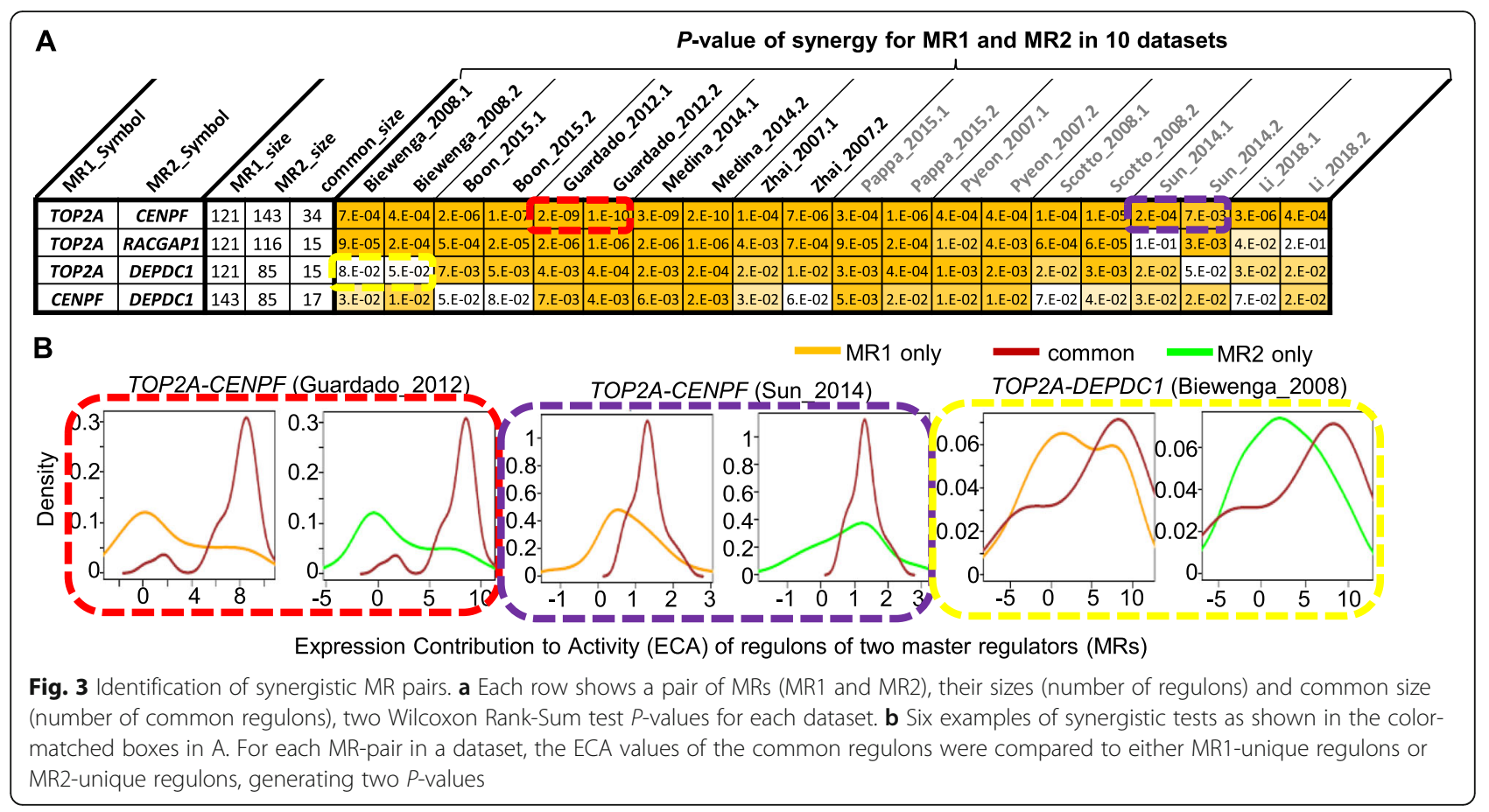

with the expression of the two genes (supplementary Figure 1, 2, supplementary Table 6).

\section{Somatic mutations associated with expression of TOP2A and CENPF}

The association of the expression of CENPF and the APOBEC mutagenesis (Fig. 5d) prompted us to find genes with somatic mutations (SMGs) associated with the expression of the two MRs. Using the TCGA data, we identified 45 SMGs forming 60 SMG-MR pairs, in which a SMG with either missense or LOF mutations associate with high or low expression of a MR (TOP2A or $C E N P F$ ) with $P<0.1$ (Wilcoxon Rank-Sum test) (Fig. 6a, supplementary Table 7). Majority (55/60) of these associations involve missense mutations. And two third (40/ 60) of the mutations are correlated with higher expression of the two MRs (supplementary Table 7). For example, missense mutations of TP53 gene are associated with higher expression of both CENPF and TOP2A $(P<$ $0.05)$ (Fig. 6b left two). Missense mutations of $M S H 2$ $(P<0.05)$ or $R B 1(P<0.1)$ are associated with higher expression of TOP $2 A$ (Fig. $6 \mathrm{~b}$ right two). Altogether missense mutations of 28 genes are associated with higher expression of CENPF or TOP2A, among them 9 are associated with higher expression of both MRs (Fig. 6a). By grouping samples based on a combined effect of all the 28 genes, samples with missense mutations in any one of these genes have significantly higher $(P=8 \mathrm{E}-7$ and 1E-9 respectively) expression of both MRs (Fig. 6c). A functional annotation analysis using DAVID showed that genes with mutations associated with higher expression of either or both the two MRs are involved in or be annotated with DNA-directed DNA polymerase (POLD1, POLE, POLQ), chromatin regulator (ATRX, $K D M 6 A, K M T 2 C, K M T 2 B, R B 1)$, DNA repair $(A T R X$, MSH2, TP53BP1, POLE, POLQ), DNA damage (ATRX, $M S H 2$, TP53BP1, POLE, POLQ), pathways in cancer (EGFR, EP300, MSH2, TP53, RB1, CTNNA1), viral carcinogenesis (EP300, DDX3X, TP53, RB1), tumor suppressor $(M S H 2, T P 53, R B 1)$ and cell cycle (EP300, TP53, RB1) (Figs. 1 g-h, Fig. 6d and a full list in supplementary Table 8).

\section{Expression of TOP2A and CENPF in CC compared to other cancer types}

Finally, we asked the question whether the two MRs (TOP2A and CENPF) are especially significant for $\mathrm{CC}$ compared to other cancer types. Using GEPIA2, a web server integrating TCGA and the GTEx data for 33 cancer types, we found that TOP2A and CENPF expression are higher in tumor than normal tissues in most of cancer types except for LAML, which showed opposite trend for both MRs. However, cervical squamous cell carcinoma and endocervical adenocarcinoma (CESC) is one of the several cancer types ( 8 and 4 for TOP2A and CENPF, respectively) which showed most significant expression increase (ANOVA $P<1 \mathrm{E}-10$ and fold change $>4$ ) in tumor compared to normal tissues (Fig. 7a). Only three other cancer types (THYM, UCEC and UCS) showed similar magnitude of expression change for both MRs. By analyzing "Bittner Multi-cancer", a multi-cancer microarray dataset using Oncomine, we observed that TOP $2 A$ and 


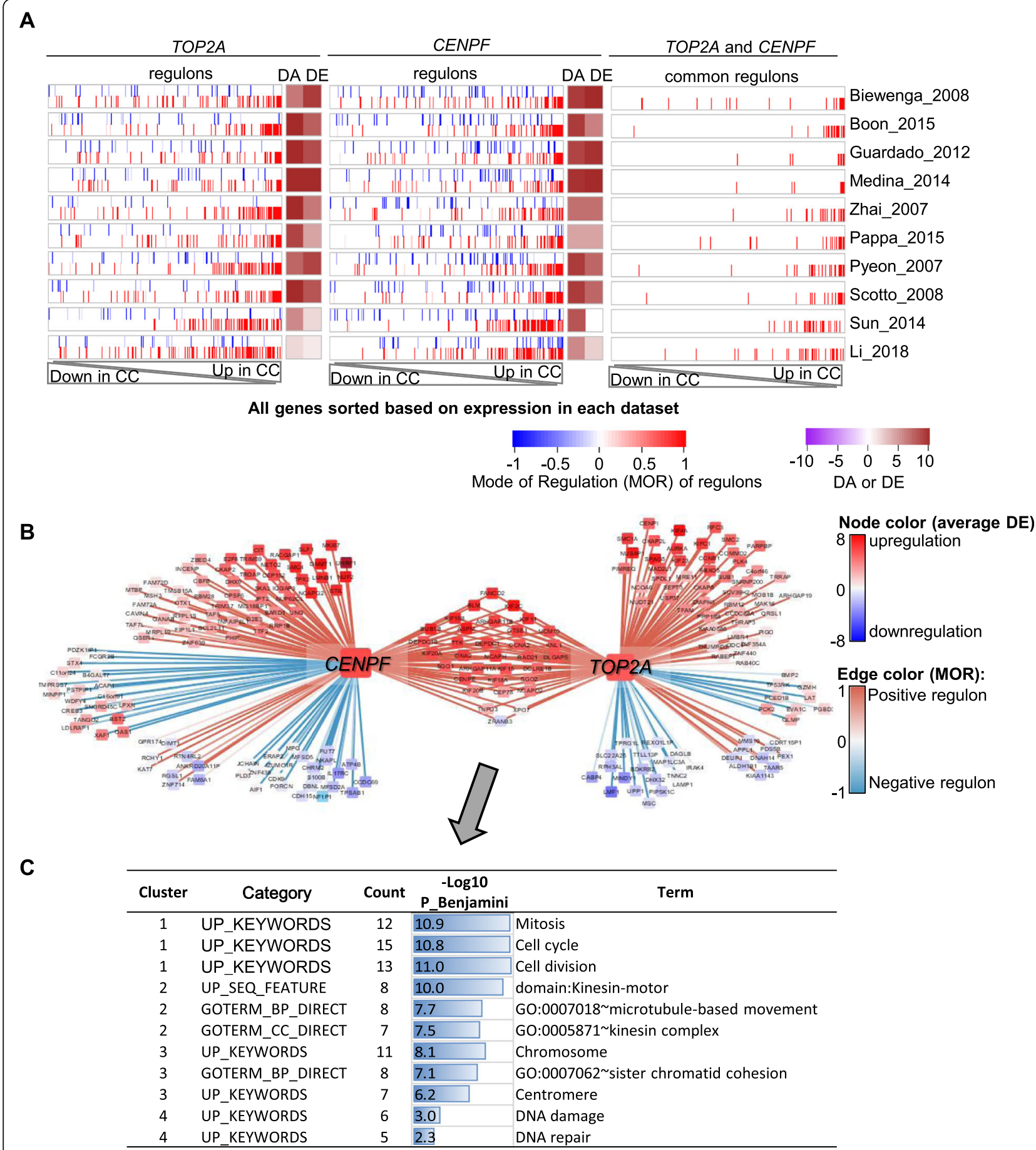

Fig. 4 Synergistic effect of TOP2A and CENPF. a Regulon enrichment plot of regulons of TOP2A (left), CENPF (middle) or common regulons (right). For each dataset, all genes were sorted based on the DE signature from most down-regulated to most up-regulated. The position of regulons in the sorted gene list was shown as a vertical blue (negative regulons) or red (positive regulons) bar. b Network view of TOP2A, CENPF and their regulons. Node color represents the average DE signature in the 10 datasets. Edge color represent the MOR of a MR and a regulon. $\mathbf{c}$ DAVID function annotation of the common regulons of TOP2A and CENPF

CENPF expression was higher (Student's $t$-test, $P=2 \mathrm{E}-7$ and $3 \mathrm{E}-4$ respectively) in $\mathrm{CC}$ than other 15 cancer types (Fig. 7b), confirming the RNA-seq data from TCGA and the GTEx.

\section{Discussion}

In current study, TOP2A-CENPF was identified as a synergistic MR pair in CC. Both MRs showed increased expression and activity in CC. TOP2A encodes DNA 


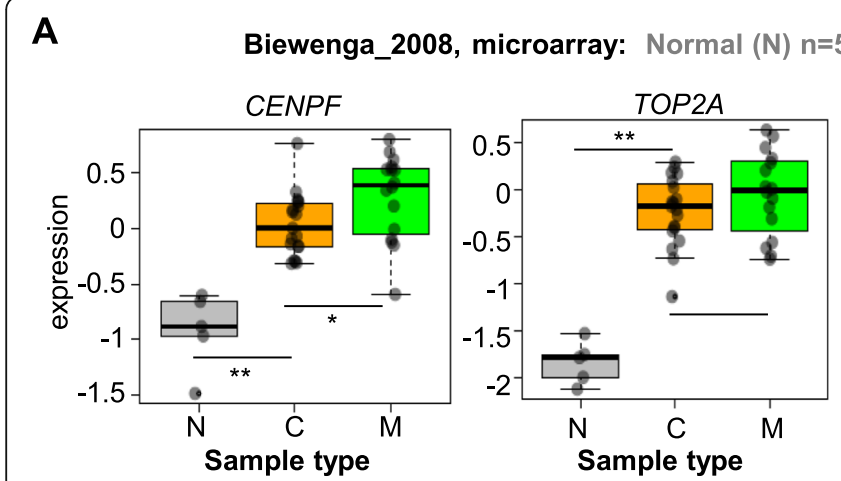

B

TCGA, RNA-seq: Normal (N) $n=3$; cervical cancer (C) $n=304$; metastasis (M) $n=2$
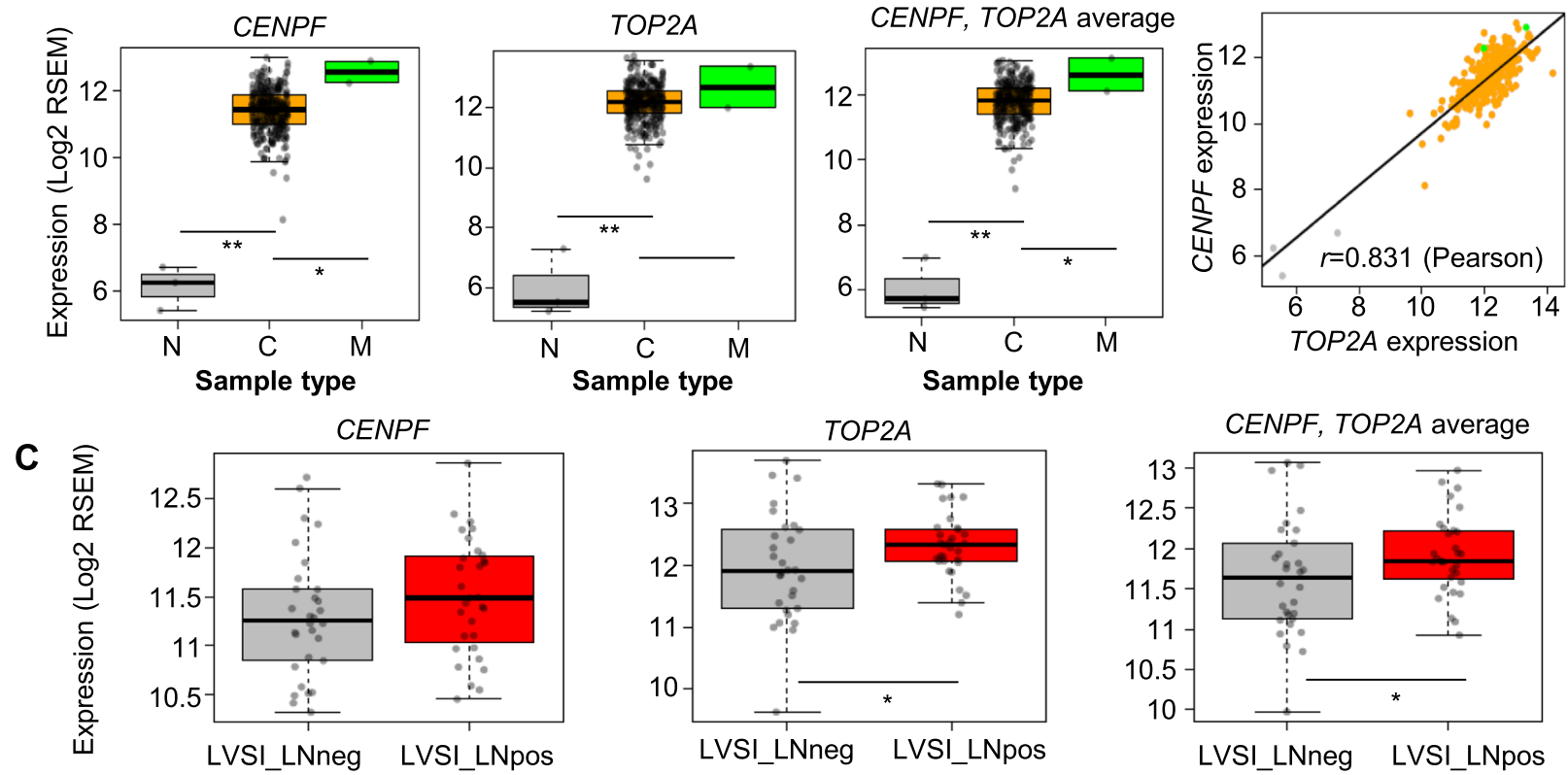

merged field based on hysterectomy, LVSI and LN

D
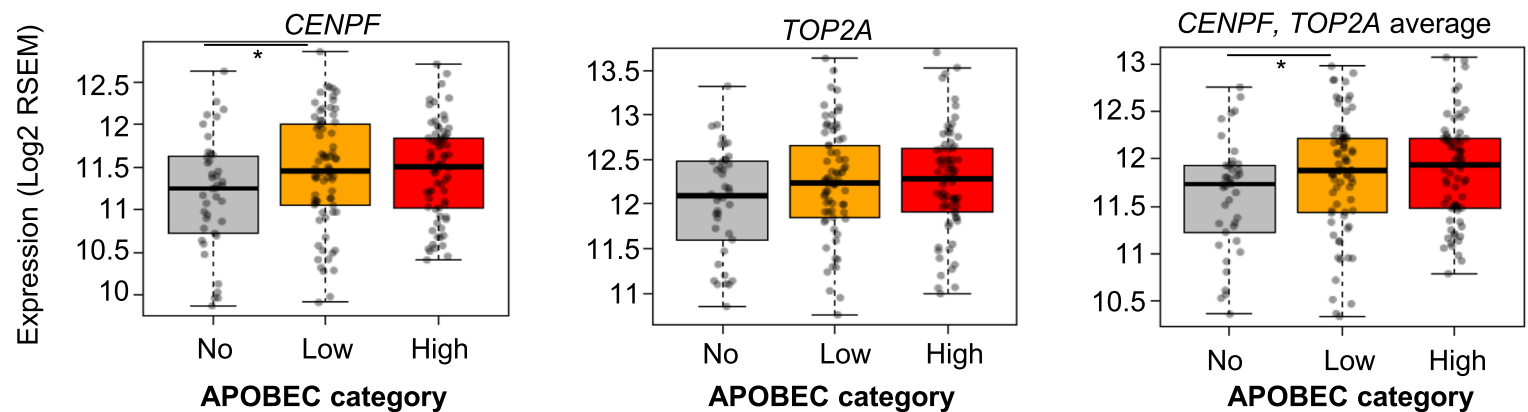

Fig. 5 TOP2A and CENPF expression is correlated with clinical or molecular features. a Left three plots, expression of CENPF, TOP2A and the average of the two for different sample types in Biewenga_2008 dataset. $P$-values were from Wilcoxon Rank-Sum test. ${ }^{*}, P<0.05 ;{ }^{* *}, P<0.01$. Right, scatter plot of the expression of the two genes. The Pearson correlation coefficient is indicated in the plot. $\mathbf{b}$ Similar to A, except that TCGA RNAseq gene expression data was used. c Similar to B, except that two clinical categories of hysterectomy, LVSI and LN (LVSI_LNneg and LVSI_LNpos) were compared. $\mathbf{d}$ Similar to B, except that three APOBEC categories were used to group samples

topoisomerase II alpha, an enzyme that controls and alters the topologic states of DNA, involves in processes such as chromosome condensation, chromatid separation, and the relief of torsional stress during DNA transcription and replication. TOP2A was frequently identified as a top overexpressed gene in CC $[26,27$, 31-33]. Unsurprisingly, TOP2A is considered a late marker in CC and immunohistochemistry or RT-PCR 


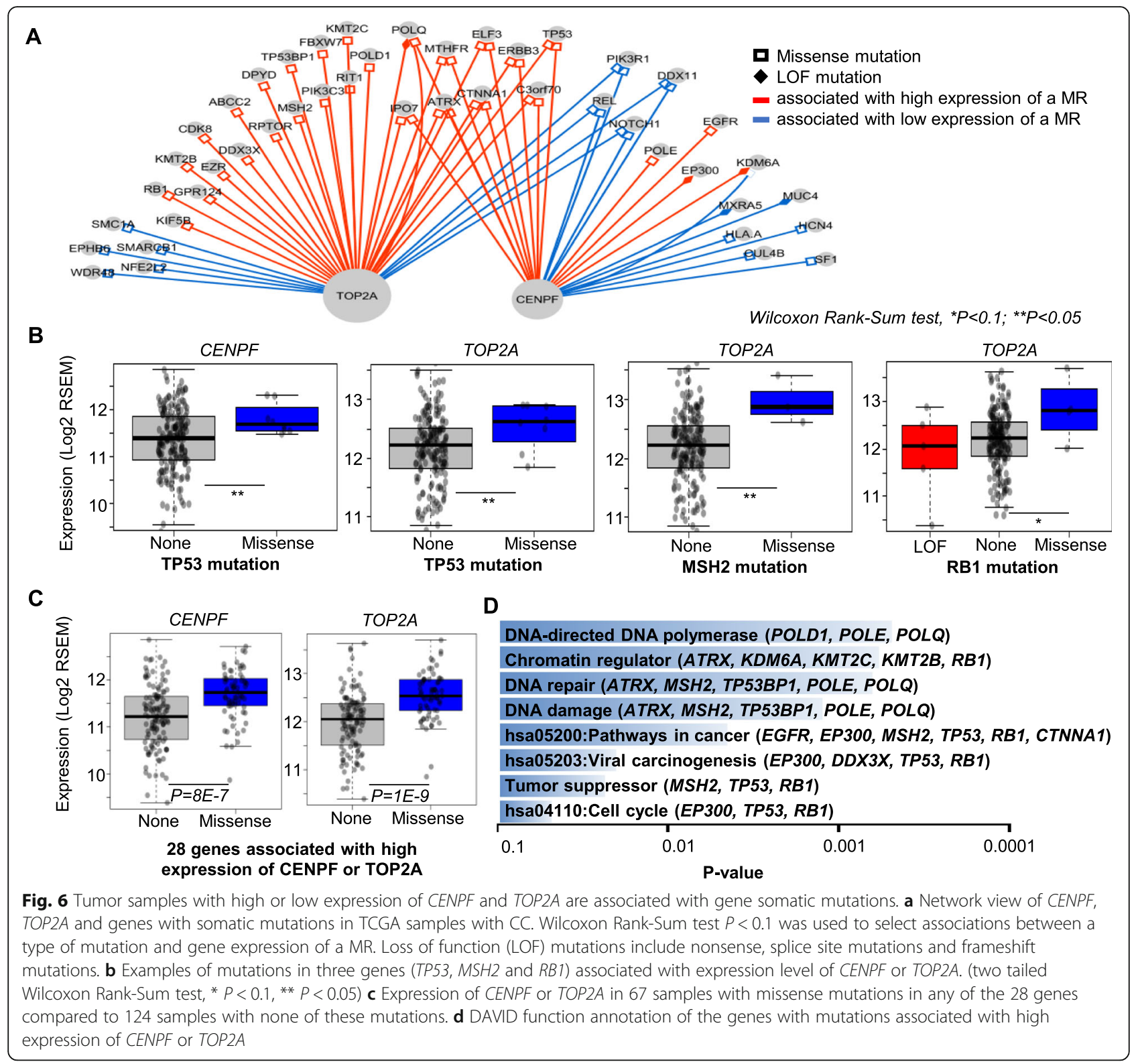

analysis of TOP $2 A$ combined with other markers (e.g. $M C M 2$, Ki-67, CDKN2A) could be used in CC screening which increases the sensitivity or specificity of the diagnosis compared to the high risk HPV test alone [34-37]. The over-expression of TOP2A seems to correlate with the grade of cervical intraepithelial neoplasia $(\mathrm{CIN})$, but does not predict prognosis in CC $[38,39]$. Consistent with these, in this study, we didn't observe any significant association between TOP $2 A$ expression and HPV status, HPV subtype, metastasis or survival among CC samples. Only LVSI and LN positive is associated with higher TOP2A expression (Fig. 5c), indicating a potential link with invasion. CENPF encodes centromere protein $\mathrm{F}$, that associates with the centromere-kinetochore complex. CENPF is a cell cycle associated nuclear antigen with maximal expression in G2/M-cells. To date, there is no reported functional role of CENPF implicated in CC. However, CENPF was reported to be overexpressed, drive tumorigenesis and/or associated with poor prognosis in several human malignancies, including breast cancer [40], prostate cancer [41-44], bladder cancer [45], gastric cancer [46], hepatocellular carcinoma [47-49], pancreatic carcinoma [50]. In this study, higher CENPF expression was found to be significantly associated with metastasis compared to CC tissues (Fig. 5a, b). Higher CENPF expression is also associated with shorter survival although the $P$-value didn't reach significance level (supplementary Figure 3). All these suggested that CENP $F$ might also be related to tumor progression to metastasis stage and predict poor prognosis in CC. Interestingly, 

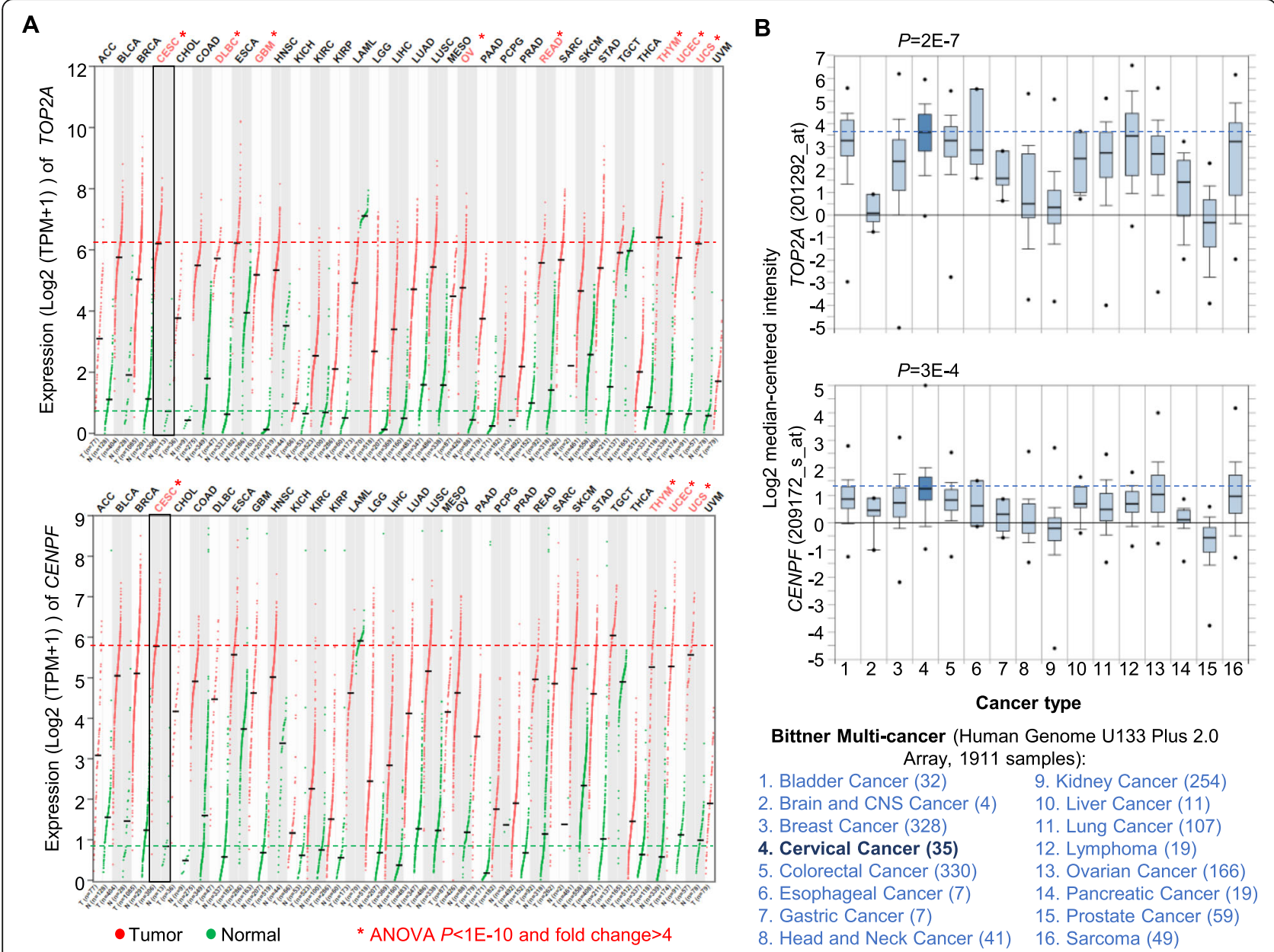

Fig. 7 Expression of CENPF and TOP2A in CC compared to other cancer types. a Expression of TOP2A and CENPF in 33 cancer types (red) and matched normal tissues (green). Data were from TCGA and GTEx analyzed using GEPIA2. Comparisons with ANOVA $P<1 \mathrm{E}-10$ and fold change $>4$ were highlighted in red text and marked with $a *$. Red and green horizontal dotted lines reflect the median value of expression for CESC and normal cervical tissue respectively. Abbreviations for cancer types are: ACC, Adrenocortical carcinoma; BLCA, Bladder Urothelial Carcinoma; BRCA, Breast invasive carcinoma; CESC, Cervical squamous cell carcinoma and endocervical adenocarcinoma; CHOL, Cholangio carcinoma; COAD, Colon adenocarcinoma; DLBC, Lymphoid Neoplasm Diffuse Large B-cell Lymphoma; ESCA, Esophageal carcinoma; GBM, Glioblastoma multiforme; HNSC, Head and Neck squamous cell carcinoma; KICH, Kidney Chromophobe; KIRC, Kidney renal clear cell carcinoma; KIRP, Kidney renal papillary cell carcinoma; LAML, Acute Myeloid Leukemia; LGG, Brain Lower Grade Glioma; LIHC, Liver hepatocellular carcinoma; LUAD, Lung adenocarcinoma; LUSC, Lung squamous cell carcinoma; MESO, Mesothelioma; OV, Ovarian serous cystadenocarcinoma; PAAD, Pancreatic adenocarcinoma; PCPG, Pheochromocytoma and Paraganglioma; PRAD, Prostate adenocarcinoma; READ, Rectum adenocarcinoma; SARC, Sarcoma; SKCM, Skin Cutaneous Melanoma; STAD, Stomach adenocarcinoma; TGCT, Testicular Germ Cell Tumors; THCA, Thyroid carcinoma; THYM, Thymoma; UCEC, Uterine Corpus Endometrial Carcinoma; UCS, Uterine Carcinosarcoma; UVM, Uveal Melanoma; b Expression analysis of TOP2A and CENPF using Bittner Multi-cancer Dataset analyzed by Oncomine. The expression in cervical cancer (CC, group 4) was compared to all other cancer types using Student's t-test. $P$-values are indicated. The blue horizontal dotted lines reflect the median value of the expression in CC

when considering the average expression of TOP2A and CENPF, both metastasis and LVSI and LN positive are significantly associated with higher average expression (Fig. 5a-c), indicating that a combined feature of the two MRs might be a better marker for CC prognosis.

According to the information from The Human Protein Atlas (http://www.proteinatlas.org) [26], both TOP2A and CENPF proteins have medium to high level of expression in most of CC tissue samples based on the immunohistochemistry results (supplementary Figure 4 and 5). These results confirmed our analysis based on RNA expression and predicted protein activity. Both of them are localized in nucleoplasm (supplementary Figure 6A-B). Both of them show cell cycle dependent expression and are expressed commonly during $\mathrm{S}$ and/or G2 phases (supplementary Figure 6C-F). These data at least make it possible that these two proteins are acting synergistically in CC cells. The localization and the cell cycle dependency of their expression are also consistent with the functions of their common regulons: e.g., cell 
cycle, DNA replication/repair, kinesin complex, chromosome etc. (Fig. 4c). A protein-protein association network analysis of TOP2A, CENPF and their common regulons also suggested a close association of these proteins (supplementary Figure 7). Although a physical interaction between TOP2A and CENPF has not been experimentally determined, many other proteins have shown such interaction. It is also possible that TOP2A and CENPF interact indirectly with each other through other proteins (supplementary Figure 7).

TOP2A and CENPF are altered (somatic mutation or copy number alternations) in only a small fraction (1 and 5\%) of CC patients (supplementary Figure 8). DNA methylation does not seem to correlate with their expression level based on analysis of $\sim 800$ cancer cell lines (supplementary Figure 9, 10). However, their high expression level is associated with CC patients with missense mutations of 28 genes (Fig. 6a, c, supplementary Table 7). Totally 67 (35\%) of the 191 samples have missense mutations in at least one of these 28 genes. And 131 (67\%) of the 191 samples have somatic mutations or copy number alternations in the 28 genes or CENPF or TOP2A (supplementary Figure 8). Many of the 28 genes are directly involved in DNA metabolism (DNA damage, DNA repair, DNA polymerase) or cell cycle. Some are involved in viral carcinogenesis, tumor suppressor and cancer pathways etc., which may eventually drive cancer progression. These data suggest that the two master regulators (TOP2A and CENPF) may "collect" the somatic mutation effect of many other genes in different cancerrelated pathways and with increased expression, promote cell proliferation and tumor progression. Persistent infections of HPV were thought to contribute to $95 \%$ of $\mathrm{CC}$ cases [51]. One mechanism of HPV to cause CC is that the viral oncogenes inactivate tumor suppressors p53 and RB, leading to increased genomic instability and accumulation of somatic mutations [51]. In this study, we found that missense mutations of TP53 and RB1 but not HPV status are associated with higher expression of TOP $2 A$ and/or CENPF (Fig. 6b, supplementary Figure 1 ). These data may suggest that the high expression of TOP2A and/or CENPF are hallmarks of CC regardless of HPV status. HPV negative CC may develop tumor through other mechanisms (e.g. accumulate mutations to inactivate TP53 or RB1). Thus, detecting TOP2A and/ or CENPF expression in clinical may decrease the false negative rate of $\mathrm{CC}$ diagnosis by HPV test alone.

We found that the two synergistic MRs TOP2A and CENPF have both the high magnitude of increase of expression (vs. normal tissue) and the high absolute expression level in CC compared to many other cancer types (Fig. 7). This may suggest that these two genes are potential anticancer drug targets in CC. The enzyme encoded by TOP $2 A$ has been designed as the target for several anticancer agents and a variety of mutations in this gene have been associated with the development of drug resistance [52]. Small molecule compounds targeting CENPF have not been reported, possibly attributed by the difficulty to target a non-enzyme target. However, reports have shown that silencing CENPF using in vitro models abolished invasion in gastric cancer [46], resulted in the cell cycle arrest at G2/M checkpoint in hepatocellular carcinoma [47], reduced levels of epithelialmesenchymal transition markers, inhibited cell proliferation, migration, and invasion, reduced global bioenergetic capacity and altered the global metabolic profiles in prostate cancer $[43,53]$. It remained to be validated whether silencing/inhibiting either $C E N P F$ or both $C E N P F$ and TOP2A will have a strong phenotypic consequence in $\mathrm{CC}$ using an in-vitro or/and in vivo model. If these targets can be validated, drugs (an antisense oligo, antibody, etc.) could be developed targeting CENPF with or without a combination of a small molecule drug targeting TOP2A in future $\mathrm{CC}$ treatment.

To explore more related to drug selection in clinical use for cancer patient, we queried CMap database using CLUE and identified potential perturbations that could reverse the over-expression signature of TOP2A, CENPF and their common regulons (supplementary Table 9). Unsurprisingly, CDK inhibitor purvalanol-a, aminopurvalanol-a, JAK3inhibitor-VI, and topoisomerase inhibitor, cell cycle inhibitor etoposide are top listed compounds $(\mathrm{CPs})$ that showed a strong negative connectivity scores in 8 cancer cell lines. The "Cell Cycle Inhibition GOF" is the top perturbation class (PCL), consistent with the function of the two MRs and their common regulons. These data may help the clinicians to select better drugs for cancer patients with an over-expression signature of the two MRs and their common regulons. This also suggests that developing or selecting drugs targeting the signal pathway they involved in might be a promising approach for $\mathrm{CC}$ and other cancer treatment.

\section{Conclusions}

In conclusion, our analysis suggested that TOP2A and CENPF are MRs that are overexpressed and activated in $\mathrm{CC}$ and synergistically regulate a common set of regulons, with functions related to cell cycle, DNA replication/repair, kinesin complex etc. Their high expression are linked to metastasis and the mutation status of a set of genes including a few tumor suppressors. They may serve as biomarkers for future $\mathrm{CC}$ diagnosis. They are also potential anticancer drug targets for future drug discovery. Our discovery could also provide guidance for clinicians to select anti-cancer drugs for CC patients. Finally, some of the observations were also observed in other cancer types, suggesting a potential wider impact of our study. 


\section{Supplementary information}

Supplementary information accompanies this paper at https://doi.org/10. 1186/s12920-020-00800-2.

Additional file 1: Supplementary Table 1: MR DA and DE signatures for the 10 datasets. "MR_set", categories of functions of MRs (TF, transcriptional factor; TFcoFac, transcriptional co-factors; SigPathway, signal pathway); "regulon_size", number of regulons for a MR; "rank_cat", category of ranking. For example, 100 indicates that the MR is a common MR of the top 100 activated MRs in the 5 discovery datasets. -100 indicates that the MR is a common MR of the top 100 suppressed MRs in the 5 discovery datasets. 0 indicates the MR is not the common top MRs in the 5 discovery datasets. "nes.", the NES score of a MR in a dataset calculated using VIPER, representing the DA signature. "pval.", the P-value of the NES from the VIPER analysis. "t.", the value representing the DE signature (e.g. the t-statistics for microarray). "DiscoverySet DA-DE consistent", whether the DA and DE are consistent. The DA-DE Consistency set was selected using |DE| value> 3 and same sign as DA in all the five discovery datasets. Supplementary Table 2: DE signatures of the common regulons of TOP2A and CENPF for the 10 datasets. "MOR_sign", sign of MOR (positive (1) or negative $(-1)$ MOR for both TOP2A-regulons and CENPFregulons). "DE.average", the average values of the DE values for the 10 datasets. Supplementary Table 3: Network of TOP2A, CENPF and their regulons. "Regulator" is the gene ID of a MR (here CENPF or TOP2A). "interaction" is always "regulate". "Target" is the regulon of a regulator. "MOR", mode of regulation, ranges between - 1 and + 1. "likelihood", range from 0 to 1 , an edge weight that indicates how strong the mutual information for an edge is. All information was extracted from the aracne.networks R package. Supplementary Table 4: "Functional Annotation Clustering" analysis of the 34 TOP2A-CENPF common regulons on DAVID 6.8 website. See DAVID website for details of methods used and interpretation of header names. "Category", the gene annotation category. UP_KEYWORDS, UniProt Keywords annotation of genes; UP_SEQ_FEATURE, UniProt sequence feature annotation of genes;

GOTERM_BP_DIRECT, Gene ontology biological process annotation; GOTERM_CC_DIRECT, Gene ontology cellular component annotation. Supplementary Table 5: CENPF and TOP2A expression in dataset Biewenga_2008 (GSE7410). The expression data were extracted from GEO database using GEO2R tool. The probe ID is indicated after the gene symbol. "Avg_Gex", the average expression value of CENPF and TOP2A. "sample_type", sample type (N, healthy cervical tissue; $C$, Early stage cervical tumour without lymph node metastasis; M, Early stage cervical tumour with lymph node metastasis). Supplementary Table 6: CENPF, TOP2A expression and clinical, molecular data in CC samples in TCGA. The clinical and molecular feature information were from the supplementary Table 1 and 2 of the publication: The Cancer Genome Atlas Research Network et al., 2017. The expression values of CENPF and TOP2A were downloaded from TSVdb. Supplementary Table 7: Somatic mutations associated with expression of CENPF and TOP2A in CC TCGA data. Each row is an association of a gene (Gene.mutation) with missense or LOF mutations and expression of CENPF or TOP2A tested using Wilcoxon Rank-Sum test. "delta. Median.Exp" is the difference of average expression of samples with mutation and samples without mutation. "Direction.Exp" indicates whether the samples with mutation has higher or lower expression than the samples without mutation. P-value is indicated in "Wilcox.P" column. Only associations with a $P<0.1$ were included in the list. Supplementary Table 8: DAVID "Functional Annotation Clustering" analysis of the genes with mutations associated with high or low expression of TOP2A or CENPF. See DAVID website for details of methods used and interpretation of header names. "Category", the gene annotation category. UP_KEYWORDS, UniProt Keywords annotation of genes; KEGG_PATHWAY, KEGG pathway annotation of genes. Supplementary Table 9: Connections of expression signature of TOP2A, CENPF and their common regulons with all expression signatures in Connectivity Map (CMap) using the QUERY app of CLUE. Each row is a perturbation in CMap. Id, perturbation ID; type, type of perturbation (CP, compound; $K D$, gene knockdown; $O E$, gene over-expression; $P C L$, perturbation class); HA1E, PC3, VCAP, A375, A549, HCC515, HT29, MCF7, HEPG2, the connectivity score for the indicated cell lines; summary, summary connectivity score of all cell lines; Average_CancerCells, average connectivity score of 8 cancer cell lines (except for HA1E (human kidney epithelial cell), all other cell lines are cancer cell lines). Name, perturbation name; Description, perturbation description; Target, the target of a compound; "belongs_to", the PCL the perturbation belongs to. The table was sorted based on ascending Average_CancerCells. The top rows represent perturbations thar reverse (downregulate) the expression of Top2A, CENPF and their common regulons. The negative connectivity scores from -95 to -100 were formatted in Excel in blue background with increasing darkness.

Additional file 2: Supplementary Figure 1. Association of CENPF and TOP2A expression with category clinical features (TCGA data). The clinical category with $>5$ samples were used for comparison. The groups were sorted by median expression from low to high. The number of samples in each group is indicated above the plot (using "\#=xx xx" format, in

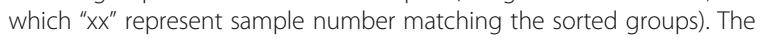
groups with the lowest and highest median expression were compared using Wilcoxon Rank-Sum test. The $P$-values are indicated on the top of the plot. $P<0.05$ was indicated by $a^{*}$. Supplementary Figure 2 . Correlation of CENPF and TOP2A expression ( $y$ axis) with continuous clinical features ( $x$ axis) (TCGA data). A linear regression line was draw. The Spearman correlation coefficient $(r)$ and $P$-value are indicated above each plot. $P<0.05$ was marked with an arrow. Supplementary Figure 3. Survival analysis of CENPF and TOP2A expression. The TCGA samples were separated to two groups (exp-high in red and exp-low in blue) using the 80th percentile of expression of CENPF or TOP2A. P-value is based on the log-rank test. Confidence intervals were shown as shaded areas. Supplementary Figure 4. Protein expression of TOP2A in CC tissues as detected by immunohistochemistry (data retrieved from The Human Protein Atlas). (A) Summary of three antibody staining results for $11 \mathrm{pa-}$ tients as detected by antibody HPA006458 or 12 patients as detected by antibodies HPA026773 and CAB002448 (full bar represents all patients). (B) An example of staining image from a patient with cervix squamous cell carcinoma. (C) An example of staining image from a patient with cervix adenocarcinoma. Supplementary Figure 5. Protein expression of CENPF in CC tissues as detected by immunohistochemistry (data retrieved from The Human Protein Atlas). (A) Summary of two antibody staining results for 11 patients per antibody (full bar represents all patients). (B) An example of staining image from a patient with cervix squamous cell carcinoma. (C) An example of staining image from a patient with cervix adenocarcinoma. Supplementary Figure 6. Protein expression in subcellular localization and cell cycle for CENPF and TOP2A (data retrieved from The Human Protein Atlas). (A) Staining of Hela cell shows that CENPF is mainly localized in nucleoplasm. (B) Staining of U2 OS cell shows that TOP2A is mainly localized in nucleoplasm. (C-D) Staining of U2 OS FUCCI cells, to characterize the cell cycle dependency of the protein expression pattern. CENPF is mostly expressed during $S$ and/or G2. (E-F) Similar to C-D unless that protein TOP2A is shown to be expressed during $S$ and/or G2 and during Mitosis. Supplementary Figure 7. ProteinProtein association network of TOP2A, CENPF and their common reguIons. Supplementary Figure 8 . OncoPrint visualization of somatic mutations, copy number alterations for the 28 genes (rows) with missense mutation associated with higher expression of CENPF and/or TOP2A in 191 cervical squamous cell carcinoma and endocervical adenocarcinoma (TCGA, Firehose Legacy) samples (columns). The mutation and gene expression profile of CENPF and TOP2A are also shown. 131 (69\%) of queried patients/samples have at least one queried gene altered. Supplementary Figure 9. Correlation of gene expression and promoter region methylation of TOP2A in 789 cancer cell lines. (A) Scatter plot, each cancer type is shown in a different color. A linear regression line is drawn for each cancer type. (B) Table of correlation coefficients and linear regression $P$-values for different cancer types. Supplementary Figure 10. Correlation of gene expression and promoter region methylation of CENPF in 823 cancer cell lines. (A) and (B) Similar to supplementary Figure 9 except that CENPF is shown.

\section{Abbreviations}

ANOVA: Analysis of variance; CC: Cervical cancer; CDS: Coding sequence; CENPF: Centromere protein F; CIN: Cervical intraepithelial neoplasia; CMap: Connectivity Map; DA: Differential activity; DAVID: The Database for Annotation, Visualization and Integrated Discovery; DE: Differential expression; ECA: Expression contribution to activity; FUCCl: Fluorescence 
Ubiquitination Cell Cycle Indicator; GEO: Gene Expression Omnibus; GEPI A2: Gene Expression Profiling Interactive Analysis 2; GO: Gene ontology; GTEx: The Genotype-Tissue Expression; HPV: Human papillomavirus; KEGG: Kyoto Encyclopedia of Genes and Genomes; LN: Lymph nodes; LOF: Loss-of-function; LVSI: Lymphovascular space invasion; MAPQ: Mapping quality score; MOR: Mode of regulation; MR: Master regulator; N: Normal tissue; NES: Normalized enrichment score; OS: Overall survival; RSEM: RNASeq by Expectation Maximization; SMG: Gene with somatic mutations; SRA: Sequence Read Archive; TCGA: The Cancer Genome Atlas; TOP2A: DNA topoisomerase II alpha; TPM: Transcript Per Million; VIPER: Virtual Inference of Protein-activity by Enriched Regulon analysis

\section{Acknowledgements}

We thank all authors listed and all the members of PZ, LC and LZ2 group for discussion and preparation of this manuscript.

\section{Authors' contributions}

$P Z, L C, L Z 2$ conceived this idea. BY, WZ, YL, YZ, YG, XT, LZ1 performed the analysis. QW, HJ, XL, HZ, PH, HY, YS, ZZ drafted the manuscript and prepared the Figs. PZ, LC, LZ2 revised the manuscript. All authors contributed to this manuscript. All authors read and approved the final manuscript.

\section{Funding}

Fees of server operation and data analysis were funded by funding from Zhejiang Province, China (Public welfare Technology Application Project, No. 2017C37046) and Medical Science and Technology Development Plan of Shandong Province (Grant No.2017WS308).

Availability of data and materials

Overall survival datasets

\begin{tabular}{llll}
\hline Data Name & Data type & Database & ID or URL \\
\hline Biewenga_2008 & Microarray & GEO & GSE7410 \\
Boon_2015 & Microarray & GEO & GSE63514 \\
Guardado_2012 & Microarray & GEO & GSE29570 \\
Medina_2014 & Microarray & GEO & GSE52903
\end{tabular}

Zhai_2007 Microarray GEO GSE7803

Li_2018 RNA-seq GEO GSE107472

Pappa_2015 Microarray GEO GSE63678

Pyeon_2007 Microarray GEO GSE6791

Scotto_2008 Microarray GEO GSE9750

Sun_2014 Microarray GEO GSE55940

human DNA UCSC genome

reference sequences browser

genome

viper

$\mathrm{R}$ package

Bioconductor

TOP2A or CENPF RSEM (RNA-Seq TSVdb gene expression by Expectation data for TCGA Maximization) samples values
(Continued)

\begin{tabular}{|c|c|c|c|}
\hline Data Name & Data type & Database & ID or URL \\
\hline $\begin{array}{l}\text { clinical and } \\
\text { molecular } \\
\text { features of CC } \\
\text { samples from } \\
\text { TCGA }\end{array}$ & $\begin{array}{l}\text { supplementary } \\
\text { table }\end{array}$ & $\begin{array}{l}\text { Cancer } \\
\text { Genome Atlas } \\
\text { Research } \\
\text { Network et al., } \\
2017\end{array}$ & $\begin{array}{l}\text { https://static-content. } \\
\text { springer.com/esm/ } \\
\text { art\%3A10.1038\%2 } \\
\text { Fnature21386/ } \\
\text { MediaObjects/415 } \\
\text { 86_2017_ } \\
\text { BFnature21386___. } \\
\text { MOESM213_ESM.zip }\end{array}$ \\
\hline
\end{tabular}

Ethics approval and consent to participate

Not applicable.

\section{Consent for publication}

Not applicable.

\section{Competing interests}

The authors declare no conflict of interest.

\section{Author details}

'Department of Laboratory, Hangzhou Jianggan District People's Hospital, Hangzhou, Zhejiang, China. 'Department of Gynecology, Xiao shan Hospital, Hangzhou, Zhejiang, China. ${ }^{3}$ Department of Gynecology, Qingdao Municipal Hospital, Qingdao, Shandong, China. ${ }^{4}$ Department of Laboratory, Jinhua People's Hospital, Jinhua, Zhejiang, China. ${ }^{5}$ Department of Laboratory, Zhejiang Jinhua Guangfu Hospital, Jinhua, Zhejiang, China. ${ }^{6}$ Medicine Reproductive Centre, Jinhua People's Hospital, Jinhua, Zhejiang, China. ${ }^{7}$ Tianjia Genomes Tech CO., LTD., Hefei, Anhui, China. ${ }^{8}$ Department of Functional Discipline, School of medicine Jinhua, Jinhua, Zhejiang, China.

Received: 12 March 2020 Accepted: 22 September 2020

Published online: 06 October 2020

\section{References}

1. Cohen PA, Jhingran A, Oaknin A, Denny L. Cervical cancer. Lancet. 2019 ; 393(10167):169-82. https://doi.org/10.1016/S0140-6736(18)32470-X.

2. Li H, Wu X, Cheng X. Advances in diagnosis and treatment of metastatic cervical cancer. J Gynecol Oncol. 2016;27(4):e43. https://doi.org/10.3802/jgo. 2016.27.e43

3. Alvarez MJ, Shen Y, Giorgi FM, et al. Functional characterization of somatic mutations in cancer using network-based inference of protein activity. Nat Genet. 2016:48(8):838-47. https://doi.org/10.1038/ng.3593.

4. Clough E, Barrett T. The gene expression omnibus database. Methods Mol Biol. 2016;1418:93-110. https://doi.org/10.1007/978-1-4939-3578-9 5.

5. Biewenga P, Buist MR, Moerland PD, et al. Gene expression in early stage cervical cancer. Gynecol Oncol. 2008;108(3):520-6. https://doi.org/10.1016/j. ygyno.2007.11.024

6. den Boon JA, Pyeon D, Wang SS, et al. Molecular transitions from papillomavirus infection to cervical precancer and cancer: role of stromal estrogen receptor signaling. Proc Natl Acad Sci U S A. 2015;112(25):E325564. https://doi.org/10.1073/pnas.1509322112.

7. Guardado-Estrada M, Medina-Martínez I, Juárez-Torres E, et al. The Amerindian mtDNA haplogroup B2 enhances the risk of HPV for cervical cancer: de-regulation of mitochondrial genes may be involved. J Hum Genet. 2012;57(4):269-76. https://doi.org/10.1038/jhg.2012.17.

8. Medina-Martinez I, Barrón V, Roman-Bassaure E, et al. Impact of gene dosage on gene expression, biological processes and survival in cervical cancer: a genome-wide follow-up study. PLoS One. 2014:9(5):e97842. https://doi.org/10.1371/journal.pone.0097842.

9. Zhai $Y$, Kuick $R$, Nan B, et al. Gene expression analysis of preinvasive and invasive cervical squamous cell carcinomas identifies HOXC10 as a key mediator of invasion. Cancer Res. 2007;67(21):10163-72. https://doi.org/10. 1158/0008-5472.CAN-07-2056.

10. Li $\mathrm{S}$, Teng $\mathrm{S}, \mathrm{Xu}$ J, et al. Microarray is an efficient tool for circRNA profiling. Brief Bioinform. 2019;20(4):1420-33. https://doi.org/10.1093/bib/bby006. 
11. Pappa Kl, Polyzos A, Jacob-Hirsch J, et al. Profiling of discrete gynecological cancers reveals novel transcriptional modules and common features shared by other Cancer types and embryonic stem cells. PLoS One. 2015;10(11): e0142229. https://doi.org/10.1371/journal.pone.0142229.

12. Pyeon D, Newton MA, Lambert PF, et al. Fundamental differences in cell cycle deregulation in human papillomavirus-positive and human papillomavirus-negative head/neck and cervical cancers. Cancer Res. 2007; 67(10):4605-19. https://doi.org/10.1158/0008-5472.CAN-06-3619.

13. Rodriguez Faba O, Palou J, Vila Reyes $\mathrm{H}$, et al. Treatment options and predictive factors for recurrence and cancer-specific mortality in bladder cancer after renal transplantation: a multi-institutional analysis. Opciones terapéuticas y factores predictivos de recurrencia y mortalidad cáncerespecífica en pacientes con tumor vesical después de trasplante renal: análisis multiinstitucional. Actas Urol Esp. 2017;41(10):639-45. https://doi.org/ 10.1016/j.acuro.2017.05.007

14. Sun NX, Ye C, Zhao Q, et al. Long noncoding RNA-EBIC promotes tumor cell invasion by binding to $\mathrm{EZH} 2$ and repressing E-cadherin in cervical cancer. PLoS One. 2014;9(7):e100340. https://doi.org/10.1371/journal.pone. 0100340.

15. Leinonen $R$, Sugawara $H$, Shumway M; International nucleotide sequence database collaboration. The sequence read archive. Nucleic Acids Res 2011; 39(Database issue):D19-D21. https://doi.org/10.1093/nar/gkq1019.

16. Dobin A, Davis CA, Schlesinger F, et al. STAR: ultrafast universal RNA-seq aligner. Bioinformatics. 2013;29(1):15-21. https://doi.org/10.1093/ bioinformatics/bts635.

17. Love MI, Huber W, Anders S. Moderated estimation of fold change and dispersion for RNA-seq data with DESeq2. Genome Biol. 2014;15(12):550. https://doi.org/10.1186/s13059-014-0550-8

18. Lachmann A, Giorgi FM, Lopez G, Califano A. ARACNe-AP: gene network reverse engineering through adaptive partitioning inference of mutual information. Bioinformatics. 2016;32(14):2233-5. https://doi.org/10.1093/ bioinformatics/btw216.

19. Shannon P, Markiel A, Ozier O, et al. Cytoscape: a software environment for integrated models of biomolecular interaction networks. Genome Res. 2003: 13(11):2498-504. https://doi.org/10.1101/gr.1239303.

20. Huang da W, Sherman BT, Lempicki RA. Systematic and integrative analysis of large gene lists using DAVID bioinformatics resources. Nat Protoc. 2009; 4(1):44-57. https://doi.org/10.1038/nprot.2008.211.

21. Tomczak K, Czerwińska P, Wiznerowicz M. The Cancer genome atlas (TCGA): an immeasurable source of knowledge. Contemp Oncol (Pozn). 2015;19(1A): A68-77. https://doi.org/10.5114/wo.2014.47136.

22. Sun W, Duan T, Ye P, et al. TSVdb: a web-tool for TCGA splicing variants analysis. BMC Genomics. 2018;19(1):405. https://doi.org/10.1186/s12864-0184775-x.

23. Cancer Genome Atlas Research Network; Albert Einstein College of Medicine; Analytical Biological Services; Integrated genomic and molecular characterization of cervical cancer. Nature. 2017;543(7645):378-84. https:// doi.org/10.1038/nature21386.

24. Tang Z, Kang B, Li C, Chen T, Zhang Z. GEPIA2: an enhanced web server for large-scale expression profiling and interactive analysis. Nucleic Acids Res. 2019:47(W1):W556-60. https://doi.org/10.1093/nar/gkz430.

25. Rhodes DR, Kalyana-Sundaram S, Mahavisno V, et al. Oncomine 3.0: genes, pathways, and networks in a collection of 18,000 cancer gene expression profiles. Neoplasia. 2007;9(2):166-80. https://doi.org/10.1593/neo.07112.

26. Thul PJ, Åkesson L, Wiking $M$, et al. A subcellular map of the human proteome. Science. 2017;356(6340)):eaal3321. https://doi.org/10.1126/ science.aal3321.

27. Gao J, Aksoy BA, Dogrusoz U, et al. Integrative analysis of complex cancer genomics and clinical profiles using the cBioPortal. Sci Signal. 2013;6(269): pl1. Published 2013 Apr 2. https://doi.org/10.1126/scisignal.2004088.

28. Szklarczyk D, Gable AL, Lyon D, et al. STRING v11: protein-protein association networks with increased coverage, supporting functional discovery in genome-wide experimental datasets. Nucleic Acids Res. 2019; 47(D1):D607-13. https://doi.org/10.1093/nar/gky1131.

29. Ghandi M, Huang FW, Jané-Valbuena J, et al. Next-generation characterization of the Cancer cell line encyclopedia. Nature. 2019;569(7757): 503-8. https://doi.org/10.1038/s41586-019-1186-3.

30. Subramanian A, Narayan R, Corsello SM, et al. A Next Generation Connectivity Map: L1000 Platform and the First 1,000,000 Profiles. Cell. 2017; 171(6):1437-52. e17. https://doi.org/10.1016/j.cell.2017.10.049.
31. Martin CM, Astbury K, McEvoy L, O'Toole S, Sheils O, O'Leary JJ. Gene expression profiling in cervical cancer: identification of novel markers for disease diagnosis and therapy. Methods Mol Biol. 2009;511:333-59. https:// doi.org/10.1007/978-1-59745-447-6_15.

32. Rajkumar T, Sabitha K, Vijayalakshmi N, et al. Identification and validation of genes involved in cervical tumourigenesis. BMC Cancer. 2011;11:80. Published 2011 Feb 22. https://doi.org/10.1186/1471-2407-11-80.

33. Wu DM, Shi J, Liu T, Deng SH, Han R, Xu Y. Integrated analysis reveals down-regulation of SPARCL1 is correlated with cervical cancer development and progression. Cancer Biomark. 2018;21(2):355-65. https://doi.org/10.3233/ CBM-170501.

34. Tornesello ML, Buonaguro L, Giorgi-Rossi P, Buonaguro FM. Viral and cellular biomarkers in the diagnosis of cervical intraepithelial neoplasia and cancer. Biomed Res Int. 2013;2013:519619. https://doi.org/10.1155/2013/519619.

35. Dixon EP, King LM, Nelson $R$, et al. Characterization and clinical validation of MCM2 and TOP2A monoclonal antibodies in the BD ProEx ${ }^{\text {TM }} \mathrm{C}$ assay: an immunoassay which detects aberrant S-phase induction in cervical tissue. J Immunol Methods. 2017;442:35-41. https://doi.org/10.1016/j.jim.2017.01.002.

36. Peres $\mathrm{AL}$, Paz E, Silva KM, de Araújo RF, et al. Immunocytochemical study of TOP2A and Ki-67 in cervical smears from women under routine gynecological care. J Biomed Sci. 2016;23(1):42. Published 2016 May 12. https://doi.org/10.1186/s12929-016-0258-z.

37. Del Pino M, Svanholm-Barrie C, Torné $\mathrm{A}$, et al. mRNA biomarker detection in liquid-based cytology: a new approach in the prevention of cervical cancer. Mod Pathol. 2015;28(2):312-20. https://doi.org/10.1038/modpathol.2014.106.

38. Branca M, Giorgi C, Ciotti M, et al. Over-expression of topoisomerase llalpha is related to the grade of cervical intraepithelial neoplasia (CIN) and highrisk human papillomavirus (HPV), but does not predict prognosis in cervical cancer or HPV clearance after cone treatment. Int J Gynecol Pathol. 2006; 25(4):383-92. https://doi.org/10.1097/01.pgp.0000209573.54457.32.

39. Smrkolj S, Erzen M, Rakar S. Prognostic significance of topoisomerase II alpha and collagen IV immunoexpression in cervical cancer. Eur J Gynaecol Oncol. 2010;31(4):380-5.

40. Sun J, Huang J, Lan J, et al. Overexpression of CENPF correlates with poor prognosis and tumor bone metastasis in breast cancer. Cancer Cell Int. 2019;19:264. https://doi.org/10.1186/s12935-019-0986-8.

41. Zhuo YJ, Xi M, Wan YP, et al. Enhanced expression of centromere protein F predicts clinical progression and prognosis in patients with prostate cancer. Int J Mol Med. 2015;35(4):966-72. https://doi.org/10.3892/ijmm.2015.2086.

42. Göbel C, Özden C, Schroeder C, et al. Upregulation of centromere protein F is linked to aggressive prostate cancers. Cancer Manag Res. 2018;10:5491504. https://doi.org/10.2147/CMAR.S165630.

43. Shahid M, Kim M, Lee MY, et al. Downregulation of CENPF remodels prostate Cancer cells and alters cellular metabolism. Proteomics. 2019;19(11): e1900038. https://doi.org/10.1002/pmic.201900038.

44. Aytes A, Mitrofanova A, Lefebvre C, et al. Cross-species regulatory network analysis identifies a synergistic interaction between FOXM1 and CENPF that drives prostate cancer malignancy. Cancer Cell. 2014;25(5):638-51. https:// doi.org/10.1016/j.ccr.2014.03.017.

45. Shi J, Zhang P, Liu L, Min X, Xiao Y. Weighted gene coexpression network analysis identifies a new biomarker of CENPF for prediction disease prognosis and progression in nonmuscle invasive bladder cancer. Mol Genet Genomic Med. 2019;7(11):e982. https://doi.org/10.1002/mgg3.982.

46. Chen EB, Qin X, Peng K, et al. HnRNPR-CCNB1/CENPF axis contributes to gastric cancer proliferation and metastasis. Aging (Albany NY). 2019;11(18): 7473-91. https://doi.org/10.18632/aging.102254.

47. Dai $Y$, Liu L, Zeng T, et al. Characterization of the oncogenic function of centromere protein $\mathrm{F}$ in hepatocellular carcinoma. Biochem Biophys Res Commun. 2013;436(4):711-8. https://doi.org/10.1016/j.bbrc.2013.06.021.

48. Ho DW, Kai AK, Ng IO. TCGA whole-transcriptome sequencing data reveals significantly dysregulated genes and signaling pathways in hepatocellular carcinoma. Front Med. 2015;9(3):322-30. https://doi.org/10.1007/s11684-0150408-9.

49. Yang X, Miao BS, Wei CY, et al. Lymphoid-specific helicase promotes the growth and invasion of hepatocellular carcinoma by transcriptional regulation of centromere protein F expression. Cancer Sci. 2019;110(7): 2133-44. https://doi.org/10.1111/cas.14037.

50. Zhou Z, Cheng Y, Jiang $Y$, et al. Ten hub genes associated with progression and prognosis of pancreatic carcinoma identified by co-expression analysis. Int J Biol Sci. 2018;14(2):124-36. https://doi.org/10.7150/ijbs.22619. 
51. Haque W, Verma V, Butler EB, Teh BS. Radical Cystectomy Versus Chemoradiation for Muscle-invasive Bladder Cancer: Impact of Treatment Facility and Sociodemographics. Anticancer Res. 2017;37(10):5603-8. https:// doi.org/10.21873/anticanres.11994.

52. Sathish M, Kavitha B, Nayak VL, et al. Synthesis of podophyllotoxin linked $\beta$ carboline congeners as potential anticancer agents and DNA topoisomerase II inhibitors. Eur J Med Chem. 2018;144:557-71. https://doi.org/10.1016/j. ejmech.2017.12.055.

53. Shahid M, Lee MY, Piplani H, et al. Centromere protein F (CENPF), a microtubule binding protein, modulates cancer metabolism by regulating pyruvate kinase M2 phosphorylation signaling. Cell Cycle. 2018;17(24):280218. https://doi.org/10.1080/15384101.2018.1557496

\section{Publisher's Note}

Springer Nature remains neutral with regard to jurisdictional claims in published maps and institutional affiliations.

Ready to submit your research? Choose BMC and benefit from:

- fast, convenient online submission

- thorough peer review by experienced researchers in your field

- rapid publication on acceptance

- support for research data, including large and complex data types

- gold Open Access which fosters wider collaboration and increased citations

- maximum visibility for your research: over $100 \mathrm{M}$ website views per year

At $\mathrm{BMC}$, research is always in progress.

Learn more biomedcentral.com/submissions 